\title{
VLT near- to mid-IR imaging and spectroscopy of the M 17 UC1 - IRS5 region ${ }^{\star}$
}

\author{
Zhiwei Chen ${ }^{1,2,3}$, Dieter E. A. Nürnberger ${ }^{2}$, Rolf Chini ${ }^{2,4}$, Zhibo Jiang ${ }^{1}$, and Min Fang ${ }^{1}$ \\ 1 Purple Mountain Observatory \& Key Laboratory for Radio Astronomy, Chinese Academy of Sciences, 2 West Beijing Road, \\ 210008 Nanjing, PR China \\ e-mail: zwchen@pmo.ac.cn \\ 2 Astronomisches Institut, Ruhr-Universität Bochum, Universitätsstrasse 150, 44801 Bochum, Germany \\ 3 University of Chinese Academy of Sciences, 100039 Beijing, PR China \\ ${ }^{4}$ Instituto de Astronomía, Universidad Católica del Norte, Avenida Angamos 0610, Casilla 1280 Antofagasta, Chile
}

Received 1 September 2014 / Accepted 16 February 2015

\begin{abstract}
Aims. We investigate the surroundings of the hypercompact $\mathrm{H}$ II region M $17 \mathrm{UC1}$ to probe the physical properties of the associated young stellar objects and the environment of massive star formation.

Methods. We use diffraction-limited near-IR (VLT/NACO) and mid-IR (VLT/VISIR) images to reveal the different morphologies at various wavelengths. Likewise, we investigate the stellar and nebular content of the region with VLT/SINFONI integral field spectroscopy with a resolution $R \sim 1500$ at $H+K$ bands.

Results. Five of the seven point sources in this region show $L$-band excess emission. A geometric match is found between the $\mathrm{H}_{2}$ emission and near-IR polarized light in the vicinity of IRS5A, and between the diffuse mid-IR emission and near-IR polarization north of UC1. The $\mathrm{H}_{2}$ emission is typical for dense photodissociation regions (PDRs), which are initially far-ultraviolet pumped and repopulated by collisional de-excitation. The spectral types of IRS5A and B273A are B3-B7 V/III and G4-G5 III, respectively. The observed infrared luminosity $L_{\mathrm{IR}}$ in the range $1-20 \mu \mathrm{m}$ is derived for three objects; we obtain $2.0 \times 10^{3} L_{\odot}$ for IRS5A, $13 L_{\odot}$ for IRS5C, and $10 L_{\odot}$ for B273A.

Conclusions. IRS5 might be a young quadruple system. Its primary star IRS5A is confirmed to be a high-mass protostellar object $\left(\sim 9 M_{\odot}, \sim 1 \times 10^{5} \mathrm{yrs}\right)$; it might have terminated accretion due to the feedback from stellar activities (radiation pressure, outflow) and the expanding $\mathrm{H}$ II region of M 17. The object UC1 might also have terminated accretion because of the expanding hypercompact $\mathrm{H}$ II region, which it ionizes. The disk clearing process of the low-mass young stellar objects in this region might be accelerated by the expanding H II region. The outflows driven by UC1 are running south-north with its northeastern side suppressed by the expanding ionization front of M 17; the blue-shifted outflow lobe of IRS5A is seen in two types of tracers along the same line of sight in the form of $\mathrm{H}_{2}$ emission filament and mid-emission. The $\mathrm{H}_{2}$ line ratios probe the properties of M $17 \mathrm{SW}$ PDR, which is confirmed to have a clumpy structure with two temperature distributions: warm, dense molecular clumps with $n_{\mathrm{H}}>10^{5} \mathrm{~cm}^{-3}$ and $T \approx 575 \mathrm{~K}$ and cooler atomic gas with $n_{\mathrm{H}} \sim 3.7 \times 10^{3}-1.5 \times 10^{4} \mathrm{~cm}^{-3}$ and $T \sim 50-200 \mathrm{~K}$.
\end{abstract}

Key words. stars: early-type - stars: individual: M17 UC1 - ISM: individual objects: M17 SW - photon-dominated region (PDR) stars: formation

\section{Introduction}

Massive stars $\left(>8 M_{\odot}\right)$ affect their surroundings as a consequence of ionizing radiation and strong stellar winds throughout their life, and as a result of metal enrichment in their fate as supernovae. However, the formation process of massive stars is still a hot, open debate. Both theoretical prediction and observational evidence suggest that massive stars most likely form via accretion of material quite similar to low-mass stars, but the involved processes may not only be scaled up (Zinnecker \& Yorke 2007, and references therein). The major difference is the energetic feedback (radiation pressure, stellar winds, and outflows), which rapidly dissipates the circumstellar envelope and consequently limits the mass growth of the central young stellar object (YSO). In addition, for high-mass YSOs the earliest evolutionary phase (protostellar phase, before reaching the zero-age

* Based on observations by the European Southern Observatory Very Large Telescope on Cerro Paranal, Chile (ESO program IDs: 281.C5027(A), 281.C-5051(A, B)). main sequence, hereafter ZAMS) is very short, as characterized by Kelvin-Helmholtz timescales of less than $10^{5}$ yrs. Thus, massive stars evolve quickly, even during their accretion phase. It is thought that the time spent in the main accretion phase might be significantly less than the main-sequence lifetime, presumably on the order of a few dynamical times of the star-forming molecular core. Therefore, to accumulate sufficiently large amounts of material during the very short timescale, accretion rates of high-mass YSOs must be much higher than those of low-mass YSOs, e.g., $\gtrsim 10^{-4} M_{\odot} \mathrm{yr}^{-1}$ (e.g., Hosokawa et al. 2010, hereafter $\mathrm{H}+10$ ) compared with $<10^{-8} M_{\odot} \mathrm{yr}^{-1}$ (e.g., Fang et al. 2013). The strong feedback from massive protostars may prevent the accretion even before the arrival on the ZAMS, if accretion rates are constantly larger than a few $10^{-3} M_{\odot} \mathrm{yr}^{-1}$. This sets an approximate upper limit for the protostars several tens of solar masses. In fact, accretion rates may vary widely with the evolution of massive protostars. Massive accretion might continue in a nonsteady fashion, which potentially allows even more massive stars to form through mass accretion $(\mathrm{H}+10)$. 
The evolution of massive protostars at these high accretion rates is still the vague feature of massive star formation, which determines the feedback on their environment. Numerical simulations show that accreting massive protostars with high accretion rates have large radii. For instance, at an accretion rate of $1 \times 10^{-3} M_{\odot} \mathrm{yr}^{-1}$, the protostellar radius may exceed $100 R_{\odot}$ at maximum (e.g., Yorke \& Bodenheimer 2008; Hosokawa \& Omukai 2009). This large a radius hence leads to low effective temperature, and very low stellar ultraviolet (UV) luminosity, which might be too low for the growth of an $\mathrm{H}$ II region around the protostar (Churchwell 2002; Keto 2007; Murphy et al. 2010). High-mass protostars are believed to precede the formation of an H II region. From the observational view, a lot of high-mass protostar candidates have high IR luminosities without observable H II regions (Kumar \& Grave 2007; Molinari et al. 2008; Grave \& Kumar 2009).

Section 2 briefly introduces the massive star-forming region M 17, and particularly summarizes the studies of the M 17 UCIRS5 region. In Sect. 3, the observations and data reduction are described. The results based on near- to mid-IR data are presented in Sect. 4. The derived properties are discussed in Sect. 5, and the conclusions are presented in Sect. 6.

\section{M17 UC1 and the southwestern photodissociation region}

At a distance of $1.98_{-0.12}^{+0.14} \mathrm{kpc}$ (Xu et al. 2011), M 17 is among the best laboratories in the Galaxy for investigating the formation of massive stars. In this paper, we report near- to mid-IR imaging and integral-field spectroscopic studies for the M 17 UC1IRS5 region (see Fig. 1), which is located just west of the arclike ionization front (IF). This region is well known because of the hypercompact H II (HCHII region M 17 UC1 (Sewilo et al. 2004), which is surrounded by a circumstellar disk (Nielbock et al. 2007, hereafter $\mathrm{N}+07$ ). The other interesting object, M 17 IRS5, is a bright IR source located 5" southwest of M $17 \mathrm{UC} 1$; in contrast to the $\mathrm{HCHII}$ region, it is not detectable at $1.3 \mathrm{~cm}$ (Chini et al. 2000). Its spectral energy distribution (SED) at IR wavelengths suggests a warmer component with a color temperature of $\sim 1000 \mathrm{~K}$, and a cooler component with a color temperature of $\sim 150 \mathrm{~K}$ (Nielbock et al. 2001, hereafter N+01). The nondetection of an associated H II region is reminiscent of the early protostellar phase when the protostar is huge with a low effective temperature and low UV luminosity, meaning that IRS 5 might be younger than UC1. However, this explanation is just one of the three plausible scenarios proposed by Kassis et al. (2002, hereafter $\mathrm{K}+02$ ). An HII region with a density higher than $3 \times 10^{5} \mathrm{~cm}^{-3}$, or a heavy dusty envelope, could also escape detection. Previous near-IR polarization studies revealed infrared reflection nebulae (IRN) associated with the two sources (Chen et al. 2012, hereafter CZ+12), which might trace potential outflows. The third bright IR source, M 17 B273, projected against the edge of the arc-shaped IF, might be a YSO too (nearIR excess, see Hanson et al. 1997), but requires a spectroscopic classification.

Besides characterizing the stellar content, SINFONI integral-field spectroscopy can also be used to investigate the diffuse nebular emission of M $17 \mathrm{SW}$, which is one of the beststudied dense photodissociation regions (PDRs) in the Galaxy. At far-IR to millimeter wavelengths, studies of molecular and atomic emission indicate that the structure of the gas is highly clumped (Stutzki et al. 1988; Stutzki \& Guesten 1990; Meixner et al. 1992; Pérez-Beaupuits et al. 2010), and supported by magnetic field rather than by thermal gas pressure
(Pellegrini et al. 2007). Temperatures of $\sim 275 \mathrm{~K}$ were found toward the IF (Brogan \& Troland 2001). One characteristic of PDRs is $\mathrm{H}_{2}$ emission originating from the collisional de-excitation of $\mathrm{H}_{2}$ molecules initially excited by UV photons, which is an important heating mechanism of dense PDRs (Sternberg \& Dalgarno 1989). Sheffer \& Wolfire (2013) reported four mid-IR pure-rotational $\mathrm{H}_{2}$ lines toward M $17 \mathrm{SW}$, which are consistent with $\mathrm{H}_{2}$ emission in high-density clumps $\left(n_{\mathrm{H}}>10^{5} \mathrm{~cm}^{-3}\right)$ embedded in an interclump atomic gas of density that is two or three orders of magnitude lower (Meixner et al. 1992; Meixner \& Tielens 1993). Nürnberger et al. (2007) reported near-IR $\mathrm{H}_{2}$ emission associated with a jet ejected by a forming high-mass protostar, which is located just $1^{\prime}$ southeast to the $\mathrm{M} 17 \mathrm{UC} 1$ - IRS5 region. In this case, $\mathrm{H}_{2}$ emission is produced by thermal emission in shock fronts.

\section{Observations and data reduction}

\subsection{SINFONI observations}

At high spatial resolution (AO supported) and middle spectral resolution $(R \sim 1500)$, we took near-IR integral field spectroscopic data of the M 17 UC1 - IRS5 region (see Fig. 1) in service mode during the nights 2008-06-08/09, 2008-09-26/27 and 2008-09-27/28, using ESO's near-IR integral field spectrograph Spectrograph for INtegral Field Observations in the Near-Infrared (SINFONI; Gillessen et al. 2005) mounted on the Cassegrain focus of the VLT Yepun at the Paranal Observatory, Chile.

Overall, we observed a sequence of four adjacent target positions (see Table 1). In all cases, AO curvature sensing was performed on the reference source M17-CEN 64 $(\mathrm{RA}=18: 20: 25.71, \mathrm{Dec}=-16: 11: 41.7, \mathrm{~J} 2000)$, which is located at the distance of about 16!.5 toward the east of IRS5 (about 12" toward the southeast of B273). For appropriate sky subtraction, a nearby empty sky position was available at a distance of about $70^{\prime \prime}-90^{\prime \prime}$ toward the west of IRS5.

In a trade-off between avoiding saturation of the spectra of the central point source and optimizing both dynamic range and field of view (FOV) for the diffuse circumstellar emission, we set up SINFONI with its 100 mas pixel scale, covering an instantaneous FOV of about $3^{\prime \prime} \times 3^{\prime \prime}$ with $64 \times 32$ pixels of 50 mas $\times 100$ mas each, together with the $H+K$ grating, which comprises the wavelength range from about $1.45 \mu \mathrm{m}$ to $2.45 \mu \mathrm{m}$ at the spectral resolution of about $1.0 \mathrm{~nm}$ (dispersion of $0.50 \mathrm{~nm} / \mathrm{pixel}$ ). The detector integration times (DITs) were set to $60 \mathrm{~s}$ (with NDIT =1), both for on-source (science) exposures and off-source (sky) exposures.

For each target position, we applied a sequence of dither offsets on a $3 \times 3$ position grid with grid spacings of 0 .' 5 , resulting in an effective FOV of about $4^{\prime \prime} \times 4^{\prime \prime}$. Because the faint circumstellar material around IRS5 is rather widespread, we took additional exposures on a $3 \times 3$ position grid with grid spacings of 2 .' 0 and 2 .' 2 , increasing the effective FOV to about 7. .' $4 \times 77^{\prime \prime} 4$. In total, we gathered 27 exposures on IRS5, six exposures on the position intermediate between IRS5 and UC1, six exposures on UC1 and 18 exposures on B273. We interleaved science exposures, with the AO loop closed, every $10 \mathrm{~min}$ with several (typically 5-6) exposures on the sky position to allow for proper sky subtraction, although void of stars and diffuse emission, with the AO loop open.

To correct for telluric features and to flux-calibrate the science exposures, each night these exposures were immediately followed by observations of a suitable telluric standard star, 
Table 1. SINFONI observation parameters.

\begin{tabular}{ccccc}
\hline \hline Target & $\begin{array}{c}\text { RA } \\
(\mathrm{J} 2000)\end{array}$ & $\begin{array}{c}\text { Dec } \\
(\mathrm{J} 2000)\end{array}$ & $\begin{array}{c}\text { Exp. time } \\
(\mathrm{s})\end{array}$ & Telluric STD \\
\hline M17-IRS5 & 182024.60 & -161139.4 & $27 \times 60$ & HIP 091126 (G2 V) \\
& 182024.71 & -161137.1 & $6 \times 60$ & HIP 094378 (B5 V) \\
M17-UC1 & 182024.82 & -161134.9 & $6 \times 60$ & HIP 094378 (B5 V) \\
M17-B273 & 182025.07 & -161133.9 & $18 \times 60$ & HIP 092470 (B2 IV) \\
\hline
\end{tabular}

applying the same strategy (auto-jitter pattern, fixed sky offset) as for the science targets and matching their airmass. The telluric standard stars used in the three observation nights are listed in Table 1.

During all three nights the observing conditions were good, with clear sky transparency and telescope guide probe seeing measurements typically in the range $0 .{ }^{\prime} 6-1{ }^{\prime \prime} 2$. The exception was that thin cirrus clouds passed through during the night 200809-27/28. Correspondingly, the achieved strehl ratios were in the range $20-40 \%$, as directly measured on the spectrally collapsed data cubes.

We obtained dark frames, lamp flats, and arcs (taken with SINFONI's internal Neon and Argon lamp for the purpose of wavelength calibration) through the SINFONI scientific calibration plan. We performed all of the basic steps of data reduction, including flat-fielding, sky subtraction, bad pixel, and atmospheric distortion correction, as well as wavelength calibration with the SINFONI data reduction pipeline. Final merging of the fully reduced and wavelength calibrated individual data cubes to one mosaic covering all science targets was performed within the SINFONI pipeline, too.

\subsection{Ancillary near- to mid-IR data}

The JHKL AO imaging was carried out in 2003 June using NAOS/CONICA (NACO; Lenzen et al. 2003; Rousset et al. 2003) on the ESO VLT at the Paranal observatory, Chile. The FOV is $27^{\prime \prime} \times 27^{\prime \prime}$ with pixel resolution of $00^{\prime} 027$. The $3 \sigma$ limiting magnitudes are $J=20.2, H=19.7, K=19.3$, and $L=15.2$. The photometry was carried out merely for the point sources within the FOV of SINFONI data (see Fig. 1). The magnitudes of these point sources were extracted based on a variety of aperture sizes on the purpose of aperture correction. The final magnitudes with aperture correction are calibrated with photometric standard stars, which are HD 110621 for the $J$-band $(J=8.91)$, HD 188112 for the $H K$-band $(H=10.78, K=10.89)$, and HD 161743 for the $L$-band $(L=7.61)$. The astrometry is adjusted by referencing the NACO point sources with the detections of the SINFONI data whose astrometry was calibrated using the 2MASS catalog. With this procedure, the relative astrometric difference between the NACO data and SINFONI data is better than $0 . ' 1$.

The TIMMI 2 mid-IR imaging was carried out in 2003 July at the ESO $3.6 \mathrm{~m}$ telescope at La Silla, Chile. The observations covered the $N 1, N 10.4$, and $Q 1$ bands with $\lambda_{\text {eff }}$ of $8.7 \mu \mathrm{m}, 10.38 \mu \mathrm{m}$, and $17.72 \mu \mathrm{m}$, respectively, and all had FOV of $55^{\prime \prime} \times 38^{\prime \prime}$ with pixel scale of 0.2 . All data are limited by diffraction with a full width half maximum (FWHM) of 0.7 .

The M 17 UC1 - IRS5 region was imaged with VLT Imager and Spectrometer for mid-InfRared (VISIR; Lagage et al. 2004) in 2006 May through the Sic filter $\left(\lambda_{\text {eff }}=11.85 \mu \mathrm{m}\right)$. The observation procedures are described in $\mathrm{N}+07$. The image is of good quality $(F W H M \approx 0,32)$ limited by diffraction, with pixel scale of 0.127 . The astrometry of the TIMMI2 and VISIR imaging data were calibrated on the basis of the NACO data. The astrometric accuracy is better than $0 . ' 1$ throughout the FOV centered on the M 17 UC1 - IRS5 region.

The spectroscopy of the $N$-band silicate absorption feature was performed with TIMMI2 at the ESO $3.6 \mathrm{~m}$ telescope at La Silla, Chile, within the same observation run of UC1 $(\mathrm{N}+07)$. The seeing was 0.7 ; the slit width was $1 . .2$.

\section{Results}

\subsection{High angular resolution near-IR imaging}

The M17 UC1-IRS5 region outlines a typical interface between an H II region and a PDR, as characterized by a prominent IF seen in our previous, lower resolution near-IR images (e.g., Hoffmeister et al. 2008; CZ+12). Our high-resolution images unveil many fine structures toward this region that were not revealed before (see Fig. 1). Part of the results had been published for $\mathrm{UC1}(\mathrm{N}+07)$. Interestingly, the two luminous infrared objects (M 17 IRS5, M 17 B273) are resolved to have more than one component in the high-resolution near-IR images. If they actually are multiple systems, one needs to review these objects because they were treated as single high-mass YSO candidates, and thus the primary's brightness would have been overestimated.

The JHKL magnitudes of the objects inside the white box are listed in Table 2. The brightest component of the IRS5 system is treated as the primary star, namely IRS5A, and followed by four other fainter companions: IRS5B, IRS5C, IRS5D, and IRS5E. Similarly, B273A is chosen as the primary star of the binary B273.

\subsubsection{Point sources with IR excess}

Figure 2 shows the $H K L$ color-color diagram of the objects from Table 2. We suggest that objects located to the right of the reddening vectors show infrared excess, which traces the circumstellar material around YSOs. The level of infrared excess somehow relates to the amount of circumstellar material (Lada \& Adams 1992), which in turn reflects the evolutionary stage of YSO. All the objects with accurate $L$-band magnitudes show infrared excess, indicating that they are YSOs. The object IRS5A, B273A, and B273B all show infrared excess resembling classical T Tauri stars. IRS5B seems to have a larger infrared excess, implying a younger evolutionary stage. IRS5C is the reddest source apart from UC1, although its infrared excess is comparable to that of IRS5B. Without proper spectral type classification, we can only establish a crude mass sequence for these point sources according to their dereddened magnitudes. However, it is impossible to extract exact extinction values for these sources solely based on their locations in Fig. 2. The close positions in the $H K L$ color-color diagram suggest that all sources except IRS5C have similar reddening. The extinction of IRS5C, however, is about three times higher. From the $H$-band magnitudes, we suggest that IRS5A is the most massive source, followed by 


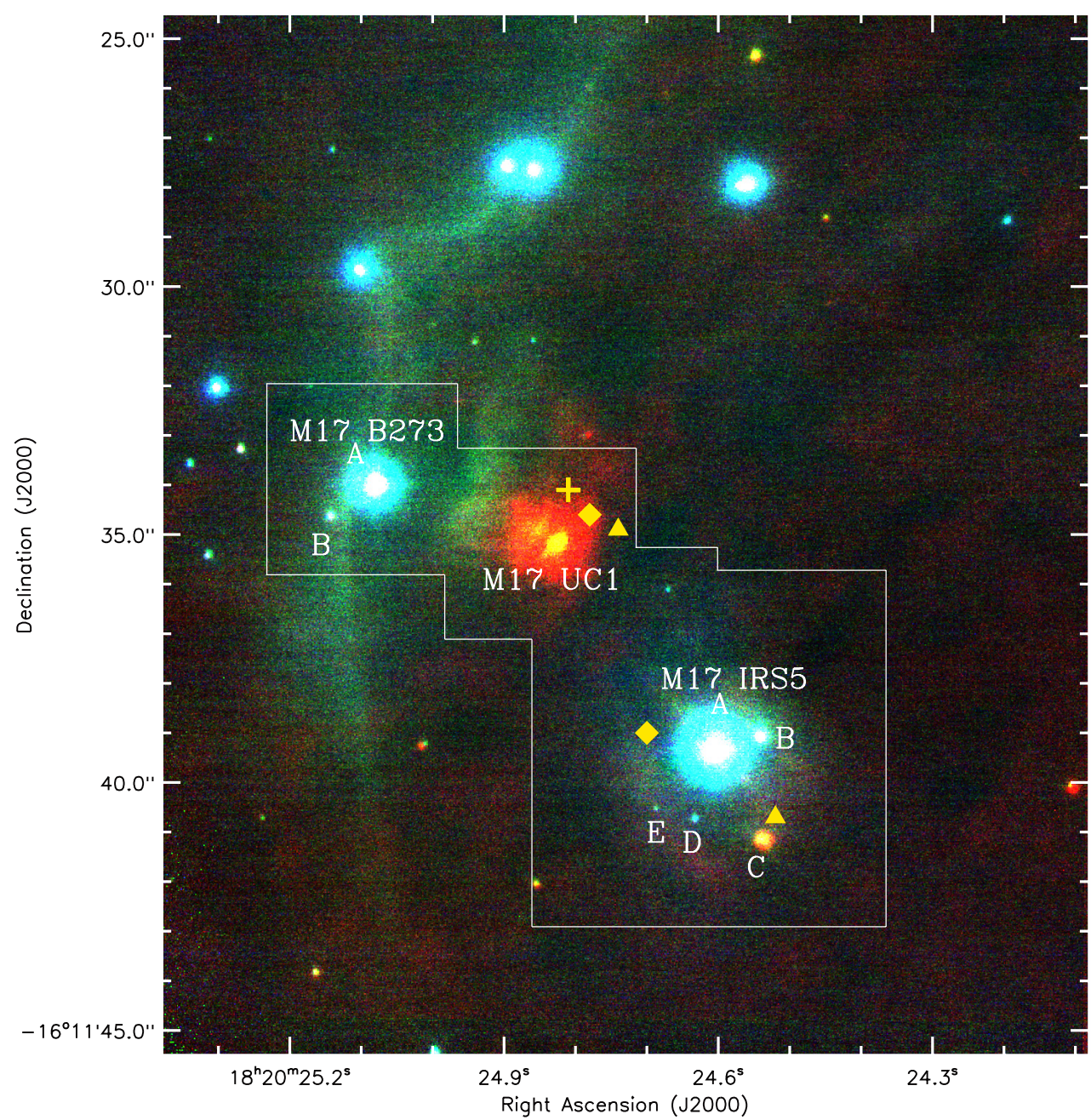

Fig. 1. Three-color image of the M 17 SW PDR taken by NACO at three near-IR broadband filters (blue: $H$; green: $K$; red: $L$ ), with the area of the SINFONI integral field spectroscopy outlined in the white box. Individual point sources discussed in this paper are labeled. Three types of maser are marked according to their coordinates: 22-GHz water masers (filled triangles, Caswell 1998; Johnson et al. 1998); Class II methanol masers at $6.66 \mathrm{GHz}$ (filled diamonds, Caswell et al. 1995, 2000); OH masers at $1.67 \mathrm{GHz}$ (thick plus, Caswell 1998).

Table 2. NACO JHKL photometry of the point sources in M 17 UC1 - IRS5 region.

\begin{tabular}{ccccccc}
\hline \hline $\begin{array}{c}\text { Source } \\
\text { ID }\end{array}$ & $\begin{array}{c}\text { RA } \\
(\mathrm{J} 2000)\end{array}$ & $\begin{array}{c}\text { Dec } \\
(\mathrm{J} 2000)\end{array}$ & $\begin{array}{c}J \\
(\mathrm{mag})\end{array}$ & $\begin{array}{c}H \\
(\mathrm{mag})\end{array}$ & $\begin{array}{c}K \\
(\mathrm{mag})\end{array}$ & $\begin{array}{c}L \\
(\mathrm{mag})\end{array}$ \\
\hline M 17 B273 & & & & & & \\
A & 182025.08 & -161134.0 & $14.03 \pm 0.01$ & $12.19 \pm 0.01$ & $10.71 \pm 0.01$ & $8.89 \pm 0.01$ \\
B & 182025.14 & -161134.6 & $20.11 \pm 0.33$ & $17.18 \pm 0.05$ & $15.26 \pm 0.04$ & $13.25 \pm 0.09$ \\
M 17 UC1 & 182024.84 & -161135.1 & - & - & $13.61 \pm 0.02$ & $6.20 \pm 0.03$ \\
M 17 IRS5 & & & & & & \\
A & 182024.60 & -161139.4 & $13.34 \pm 0.01$ & $11.38 \pm 0.02$ & $9.81 \pm 0.01$ & $8.16 \pm 0.03$ \\
B & 182024.54 & -161139.0 & $19.07 \pm 0.22$ & $15.09 \pm 0.02$ & $13.81 \pm 0.02$ & $11.56 \pm 0.03$ \\
C & 182024.54 & -161141.2 & - & $19.13 \pm 0.13$ & $14.65 \pm 0.03$ & $9.69 \pm 0.01$ \\
D & 182024.63 & -161140.7 & - & $17.97 \pm 0.12$ & $16.32 \pm 0.07$ & $15.27 \pm 0.40$ \\
E & 182024.69 & -161140.5 & - & $19.69 \pm 0.32$ & $17.75 \pm 0.18$ & - \\
\hline
\end{tabular}

Notes. Coordinates of point sources adopted here are a compromise between the astrometry of NACO and SINFONI data, whose differential has been checked at less than 0.1 throughout the FOV.

B273A and IRS5C. IRS5E has the lowest mass, while the other sources have masses in between IRS5A and IRS5E.

The area enclosed by the white outline harbors several types of maser, such as water $\left(\mathrm{H}_{2} \mathrm{O}\right)$ maser at $22 \mathrm{GHz}$, hydroxyl $(\mathrm{OH})$ maser at $1.665 \mathrm{GHz}$, and Class II methanol maser at $6.66 \mathrm{GHz}$. This variety of masers is commonly found toward regions of massive star formation (e.g., Fontani et al. 2010; Breen et al. 2010). Particularly, the Class II methanol maser is believed to be radiatively pumped, and typically coincides in position with hot molecular cores, UCH II regions, OH masers, and near-IR sources (Fontani et al. 2010). Note that $\mathrm{H}_{2} \mathrm{O}, \mathrm{OH}$, and 6.66-GHz methanol masers are all detected in the close vicinity of $\mathrm{UC} 1$, 


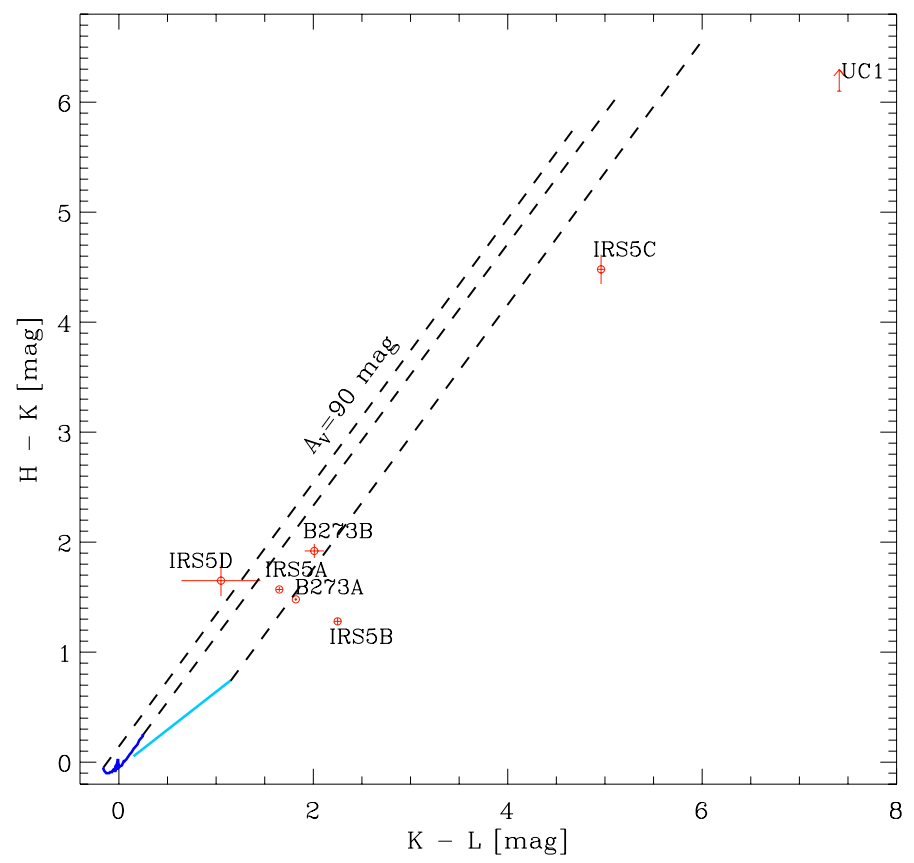

Fig. 2. NACO $K-L$ versus $H-K$ color-color diagram. The blue curve denotes the intrinsic colors of main-sequence stars (Ducati et al. 2001); the cyan line corresponds to the locus of T Tauri stars (Meyer et al. 1997). Reddening vectors (dashed lines) with slope of $E(H-K) / E(K-$ $L)=1.2$ (Hoffmeister et al. 2008) are drawn. Their lengths equal to visual extinction of $90 \mathrm{mag}$.

which coincides with the expectation of a massive forming star. Moreover, a 6.66-GHz methanol maser is detected in close proximity to IRS5A. The $\mathrm{H}_{2} \mathrm{O}$ maser at $22 \mathrm{GHz}$ is closer to IRS5C than to IRS5A. If the 22- $\mathrm{GHz} \mathrm{H}_{2} \mathrm{O}$ maser is associated with IRS5C, it might be a high-mass YSO too. The other possibility is that the $22-\mathrm{GHz} \mathrm{H}_{2} \mathrm{O}$ maser is associated with IRS5A, coexisting with the 6.66- $\mathrm{GHz}$ methanol maser.

\subsubsection{Diffuse emission}

A bright rim crossing between the B273 binary is clearly seen in Fig. 1. This rim is a portion of the IF in M 17 seen in larger view (e.g., $\mathrm{CZ}+12$ ) that represents the boundary between the $\mathrm{H}$ II region (to the northeast) and M $17 \mathrm{SW}$ (to the southwest). We note an elongated, bar-like emission feature $2^{\prime \prime}$ west to the IF. This emission bar is nearly parallel to the IF, and shows similar brightness and color with the IF. However, the emission bar has a smooth boundary while the IF is very sharp. Hence, the emission bar might not have the same physical origin as the IF.

The near-IR polarization studies toward M $17(\mathrm{CZ}+12)$ revealed a bar-like feature enhanced in $K$-band polarized light lying west to the IF. Figure 3 shows the comparison between $K$-band polarization and $\mathrm{NACO} / K$ image. The polarization pattern centered on IRS5A had been discussed in $\mathrm{CZ}+12$. The high polarization degree of $\mathrm{UC} 1$ is consistent with its two reflection lobes $(\mathrm{N}+07)$. At the position of the emission bar seen in $\mathrm{NACO} / K$ image, a concentration of polarization vectors $\sim 10 \%-15 \%$ marks the location of an IRN. From the pattern of these polarization vectors, UC1 is the best candidate for the illuminating source of the IRN. In fact, the coincidence the two bar-like features seen in different manners indicates a mass concentration of gas and dust associated with UC1 in a bar-like shape.

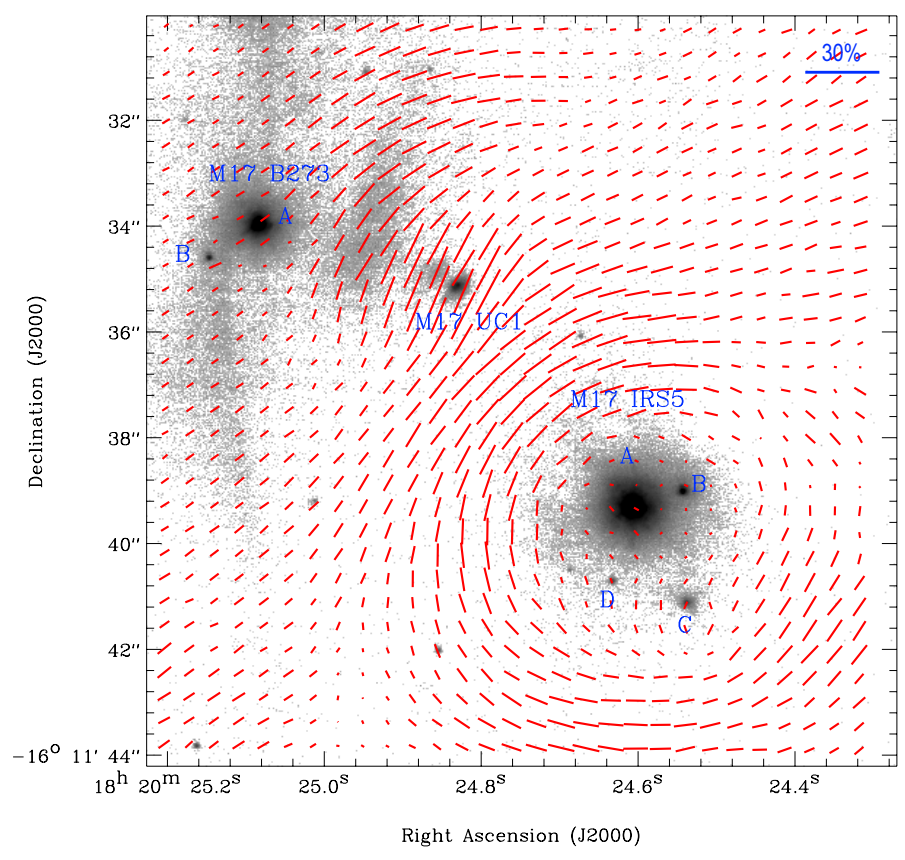

Fig. 3. IRSF $/ K$ polarization vectors (red lines; $\mathrm{CZ}+12$ ) overlaid on $\mathrm{NACO} / K$ image (grey scale).

\subsection{Mid-IR imaging}

Previous mid-IR imaging showed round morphologies both for $\mathrm{UC} 1$ and IRS5 with an angular resolution at $1-2^{\prime \prime}$. Interestingly, the new VISIR image at $11.85 \mu \mathrm{m}$ with angular resolution $\approx 0.3$ reveals substructures seen in Fig. 4 for both objects. The IF is still visible at this wavelength, but much fainter than in the $K$-band.

UC1 shows an elongated feature toward the northwest, which agrees well with its circumstellar disk orientated at PA (from north to east) $\approx 146^{\circ}$ (shown in yellow dashes in Fig. 4). Moreover, the two parts separated by the circumstellar disk are asymmetric, with the northeastern part being more extended. This asymmetric structure can be explained by an inclined circumstellar disk with angle $\approx 30^{\circ}$ with respect to the line of sight (LOS), and is proposed by $\mathrm{N}+07$ to reproduce the $K$-band asymmetric structure, which possesses a much brighter southwestern lobe. Besides the mid-IR emission in the close vicinity of UC1, it is more worthwhile to note another structure extending to north. Interestingly, this structure is spatially coinciding with the IRN discussed in Sect. 4.1.2, and fully covers the area of substantial $K$-band polarization. Conversely, the bar-like feature seen in $K$-band covers only a portion of the IRN.

The high-sensitivity VISIR image shows two lobes separated by a dark lane orientated at $\mathrm{PA} \approx 30^{\circ}$ for IRS5A. Unlike UC1, the $K$-band polarization level is very small in the bulk area of IRS5A. In addition, the $K$-band polarization found in east and south of IRS5A has no mid-IR counterparts; only the mid-IR feature in northeast of IRS5A coincides with $K$-band polarization.

Besides the two bright objects mentioned above, B273A and IRS5C are also visible in the VISIR $11.85 \mu \mathrm{m}$ image; IRS5C is even brighter than B273A at this wavelength.

In addition, the mid-IR images at other wavelengths taken by TIMMI 2 show only the two bright mid-IR objects, UC1 and IRS5A, because of the lower angular resolution and sensitivity. Thus, these mid-IR images taken by TIMMI2 are not shown here, but only the flux densities of UC1 and IRS5A are listed in Table 3. 
Table 3. Mid-IR flux densities of the YSOs in the M 17 UC1 - IRS5 region.

\begin{tabular}{lccccc}
\hline \hline Object & $\begin{array}{c}8.7 \mu \mathrm{m}^{a} \\
(\mathrm{mJy})\end{array}$ & $\begin{array}{c}10.38 \mu \mathrm{m}^{a} \\
(\mathrm{mJy})\end{array}$ & $\begin{array}{c}11.85 \mu \mathrm{m} \\
(\mathrm{mJy})\end{array}$ & $\begin{array}{c}17.72 \mu \mathrm{m}^{a} \\
(\mathrm{mJy})\end{array}$ & $\begin{array}{c}20.6 \mu \mathrm{m}^{c} \\
(\mathrm{mJy})\end{array}$ \\
\hline M17 B273A & - & - & $46 \pm 22^{b}$ & - & - \\
M 17 UC1 & $18700 \pm 1300$ & $7300 \pm 1000$ & $31300 \pm 1100^{a}$ & $146700 \pm 29700$ & $128500 \pm 6800$ \\
M 17 IRS5A & $3200 \pm 1600$ & $6800 \pm 1500$ & $9700 \pm 1100^{a}$ & $130000 \pm 31000$ & $103900 \pm 5500$ \\
M17 IRS5C & - & - & $256 \pm 25^{b}$ & - & - \\
\hline
\end{tabular}

Notes. ${ }^{(a)} 2{ }^{\prime \prime} 0$ aperture. ${ }^{(b)} 0.4$ aperture. ${ }^{(c)} 3$ '.2 aperture from $\mathrm{K}+02$.

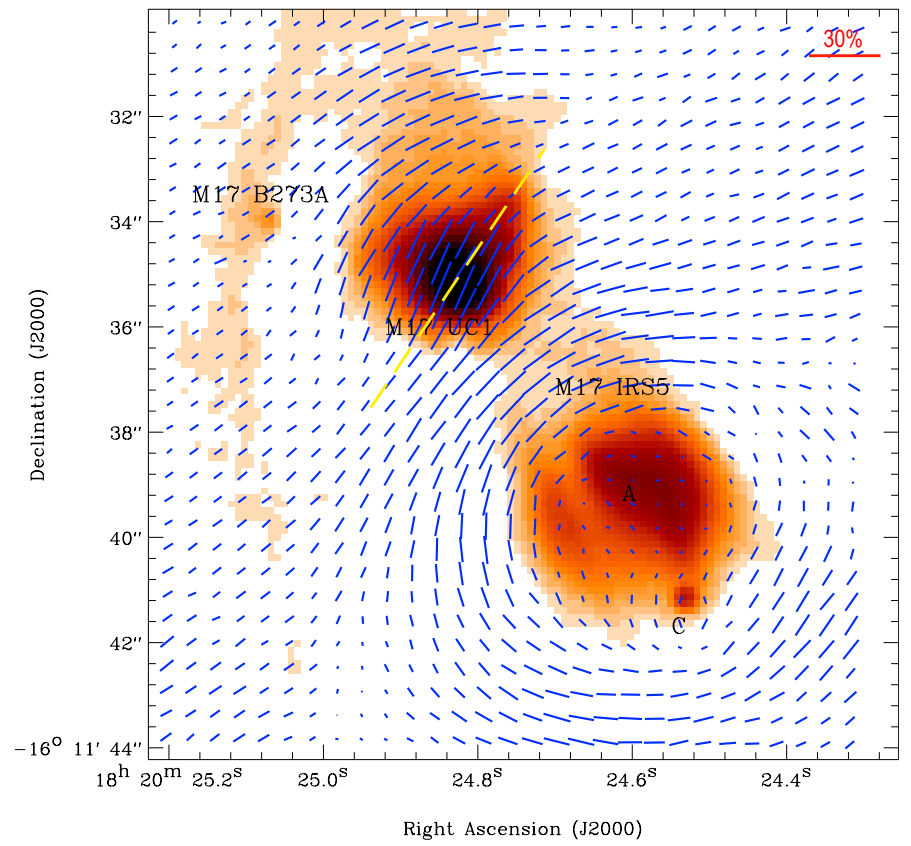

Fig. 4. VISIR $11.85 \mu \mathrm{m}$ image of the M 17 UC1-IRS5 region. IRSF/ $K$ polarization vectors (blue lines; $\mathrm{CZ}+12$ ) are overplotted as well. The disk position angle is denoted by the yellow dashes with PA of $156^{\circ}$ $(\mathrm{N}+07)$.

\subsection{SINFONI near-IR spectroscopy of diffuse content}

The SINFONI observations are centered on IRS5, and are extended northeast to cover UC1 and B273. Figure 5 shows the three-color composite of three lines. This figure reveals both the stellar content and diffuse emission in that area. For each object visible in Fig. 5, a SINFONI $H+K$ spectrum is available. However a spectral classification could only be obtained for IRS5A and B273A (Sect. 4.4), while the signal-to-noise ratio $(\mathrm{S} / \mathrm{N})$ of the other spectra was not sufficient. The $\operatorname{Br} \gamma$ and $2.059 \mu \mathrm{m}$ He I $2^{1} \mathrm{P}-2^{1} \mathrm{~S}$ line emissions are detected throughout the FOV. The location of the H II region and the IF is traced by the very strong $\mathrm{Br} \gamma$ emission at the top-left corner. On the other hand, the PDR is characterized by $2.122 \mu \mathrm{m} \mathrm{H}_{2} 1-0 \mathrm{~S}(1)$ emission. We note $\mathrm{H}_{2}$ emission surrounding IRS5A and $\mathrm{H}_{2}$ emission, coinciding with the bar-like IRN illuminated by UC1.

\subsubsection{Nebular emission lines}

The spectra in the three selected regions are shown in Fig. 6, and the observed lines are listed in Table 4. The hydrogen recombination lines such as Brackett series and $\mathrm{Pa} \alpha$ are visible in all regions. Besides the prominent atomic hydrogen lines, five atomic helium lines are also observed; among them $2.059 \mu \mathrm{m}$

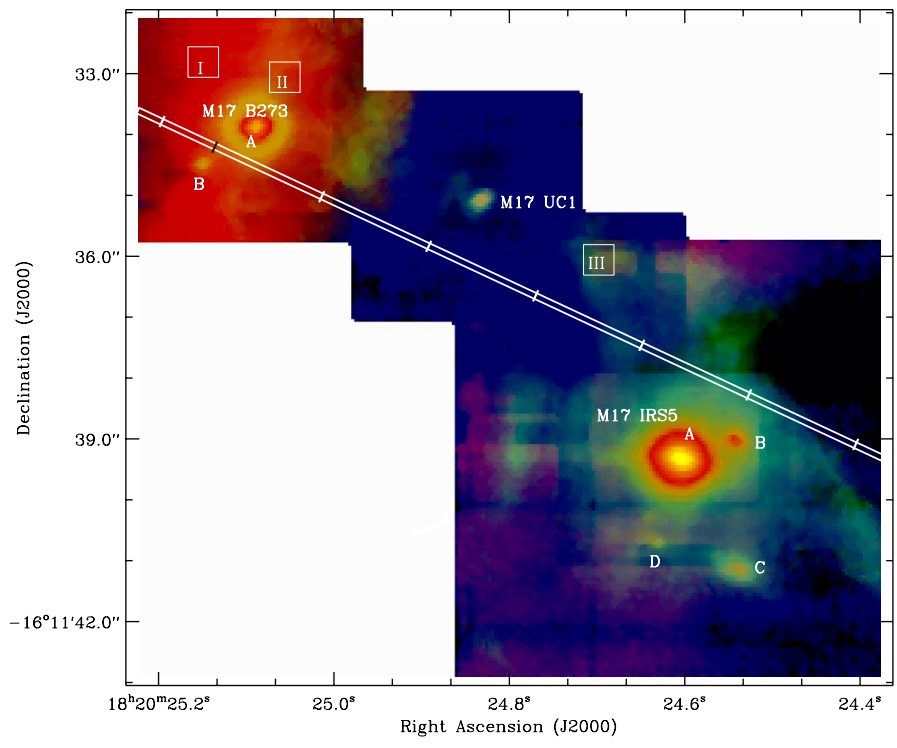

Fig. 5. Three-color image created from the emission (line+continuum) of three near-IR lines (red: $\mathrm{Br} \gamma 2.167 \mu \mathrm{m}$, green: $\mathrm{H}_{2} 1-0 \mathrm{~S}(1) 2.122 \mu \mathrm{m}$, blue: He I $2.059 \mu \mathrm{m}$ ). The three squares (I, II, and III) mark selected positions representing $\mathrm{H}$ II region, ionization front, and PDR. A strip line with tick marks is also drawn perpendicular to the IF of M $17 \mathrm{SW}$. Offset at each tick mark is $-1,0,+2,+4,+6,+8,+10,+12$ in arcsec from northeast to southwest, respectively.

He I is the strongest. Molecular hydrogen emission lines longward $2 \mu \mathrm{m}$ are also observed in region II and III. An emission feature at $2.287 \mu \mathrm{m}$ is generally classified as $\mathrm{H}_{2} v=3-2 \mathrm{~S}(2)$ line or unidentified (UID) line (e.g., Lumsden et al. 2001). However, the $\mathrm{H}_{2} / \mathrm{UID}$ line might have been mismatched for the $\mathrm{H}_{2} v=3-2 \mathrm{~S}(2)$ line because $\mathrm{H}_{2}$ emission is not spatially coincident with this $\mathrm{H}_{2} / \mathrm{UID}$ line in planetary nebulae (e.g., NGC 7027, Okumura et al. 2004). The greatest potential carrier of this UID line is [Se IV] at $2.287 \mu \mathrm{m}$, which is typically detected in highly excited ISM such as planetary nebulae and UCH II regions with hot O-type stars (Blum \& McGregor 2008, and references therein). The ionizing sources (O4 binary) of M $17 \mathrm{H}$ II region are very likely to produce the $2.287 \mu \mathrm{m}$ Se IV line in M $17 \mathrm{SW}$. The $2.287 \mu \mathrm{m}$ UID line maintains roughly the same strength throughout the FOV of Fig. 5, indicating that this line traces the ionized gas region. Therefore, we attribute the $2.287 \mu \mathrm{m}$ line to [Se IV].

\subsection{2. $\mathrm{HI} / \mathrm{H}_{2}$ transition zone of $\mathrm{M} 17 \mathrm{SW}$}

The coexistence of $\mathrm{Br} \gamma$ and $\mathrm{H}_{2} 1-0 \mathrm{~S}(1)$ in region II and III implies that region II and III may be part of the $\mathrm{HI} / \mathrm{H}_{2}$ transition zone of M $17 \mathrm{SW}$. To investigate the scale size of this $\mathrm{HI} / \mathrm{H}_{2}$ transition zone, Fig. 7 shows line strength variations of $\mathrm{Br} \gamma$, 


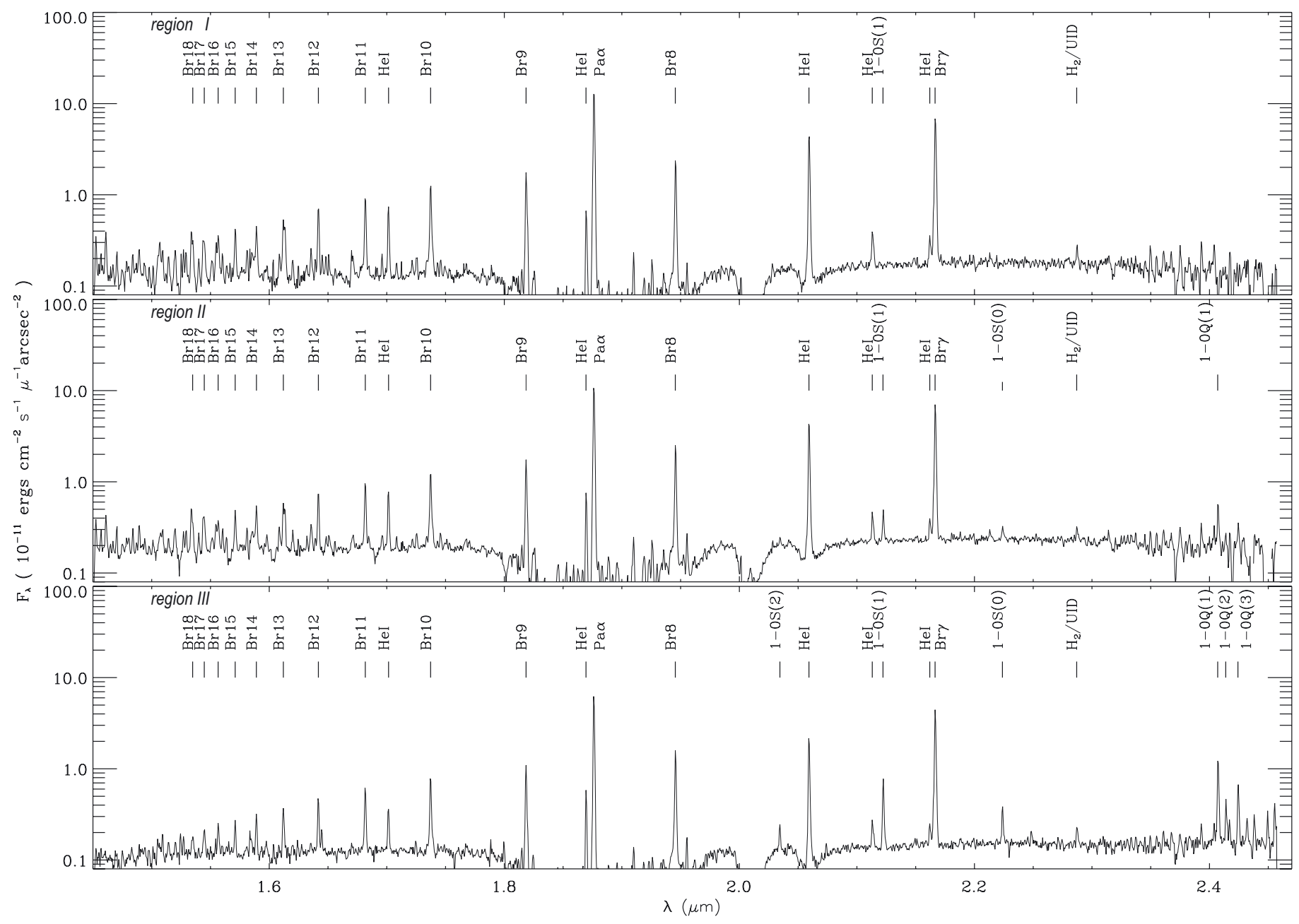

Fig. 6. Ambient spectra of the three selected positions marked as squares in Fig. 5. The telluric feature in the range $1.8-2.1 \mu \mathrm{m}$ is not corrected because its strength is in proportion to the nebular continuum, which is much weaker than the emission lines. Thus the near-IR emission lines are little affected by the telluric feature.

$2.059 \mu \mathrm{m} \mathrm{He}$, and $\mathrm{H}_{2} 1-0 \mathrm{~S}(1)$ along the strip line denoted in Fig. 5. Peaks of $\mathrm{Br} \gamma$ and $2.059 \mu \mathrm{m} \mathrm{He} \mathrm{I}$ both occur around IF. Specifically, the $2.059 \mu \mathrm{m}$ He I peak occurs $\sim 0$.' 1 closer to the $\mathrm{H}$ II region than the $\mathrm{Br} \gamma$ peak, which defines the IF. Molecular hydrogen starts to appear at offset $\sim+0$.' 4 , closer to the PDR. Along the strip line, the line strengths of $\operatorname{Br} \gamma$ and $2.059 \mu \mathrm{m}$ He I show almost identical variations, which both experience fast growth until the IF, rapid drop (offset <+0.'5), shallow decline (offset between $+0.5-+2^{\prime \prime}$ ), and roughly constant baseline (offset longwards $\left.+2^{\prime \prime}\right)$. The $\mathrm{H}_{2} 1-0 \mathrm{~S}(1)$ line strength has another type of variation, which shows four peaks along the strip line. The coexistence of atomic gas $(\mathrm{HI}, \mathrm{HeI})$ and molecular gas $\left(\mathrm{H}_{2}\right)$ in the range $+0.4-+11^{\prime \prime} .7$ and the trend further into the PDR indicates that the $\mathrm{HI} / \mathrm{H}_{2}$ transition zone of $\mathrm{M} 17 \mathrm{SW}$ almost starts from the IF and extends toward the cloud core with a projected scale size of more than $12^{\prime \prime}(0.12 \mathrm{pc})$. However, one has to be cautious because the emission from the HII region and the PDR may partly overlap along the LOS if the configuration is not strictly seen edge-on; the $\mathrm{H}$ II region emission lines originate from the surface of the PDR, while PDR emission lines form inside the PDR. In this case, the co-existence of emission lines like in Fig. 7 will not tell us the true story of the $\mathrm{HI} / \mathrm{H}_{2}$ transition zone. Comparison with a strictly edge-on PDR will help to clarify whether this scale size of $\mathrm{HI} / \mathrm{H}_{2}$ transition zone in $\mathrm{M} 17$ SW is reliable or not.
The Orion Bar is a dense PDR like M17 SW, but nearly edge-on (Allers et al. 2005, and references therein). We measured the scale size of $\mathrm{HI} / \mathrm{H}_{2}$ transition zone in the Orion Bar according to Fig. 3 of Hayashi et al. (1985) to be around 20", which is consistent with the distributions of $\mathrm{H}_{2}$ rotational lines (Allers et al. 2005). Because of the edge-on configuration of the Orion Bar, the scale size measured above is identical to the real scale size of the $\mathrm{HI} / \mathrm{H}_{2}$ transition zone, i.e., $0.044 \mathrm{pc}$ at the distance of Orion (450 pc; Hoogerwerf et al. 2000). However, the $\mathrm{HI} / \mathrm{H}_{2}$ transition zone in $\mathrm{M} 17 \mathrm{SW}$ is at least three times Orion Bar in size, which can not be simply explained by the differential properties between the two PDRs. We speculate that the presence of the three emission lines in Fig. 7 is simply a result of geometric projection. Unlike the Orion Bar, M 17 SW is indeed inclined from the LOS with a substantial angle. This result confirms the conclusion of a recent study about the LOS structure of $\mathrm{M} 17 \mathrm{SW}$ based on $\mathrm{H}_{2}$ rotational emission (Sheffer \& Wolfire 2013).

\subsection{3. $\mathrm{H}_{2}$ excitation}

As seen in Table 4, six $\mathrm{H}_{2}$ emission lines are detected. The two most prominent $\mathrm{H}_{2}$ lines are $\mathrm{H}_{2} 1-0 \mathrm{Q}(1)$ at $2.407 \mu \mathrm{m}$ and $\mathrm{H}_{2} 1-0 \mathrm{~S}(1)$ at $2.122 \mu \mathrm{m}$. The $\mathrm{H}_{2}$ emission lines in PDR are generally thought to have two physical mechanisms: fluorescent 
Table 4. Observed near-IR lines toward the M 17 UC1 - IRS5 region.

\begin{tabular}{|c|c|c|c|c|c|c|}
\hline \multirow{2}{*}{$\frac{\lambda(\mu \mathrm{m})}{\text { Obs. }}$} & \multirow[t]{2}{*}{ IDs } & \multirow[t]{2}{*}{ Transition } & \multirow[t]{2}{*}{ Eupper $(\mathrm{K})^{a}$} & \multicolumn{3}{|c|}{$F \pm \delta F\left(10^{-15} \mathrm{erg} \mathrm{cm}^{-2} \mathrm{~s}^{-1} \operatorname{arcsec}^{-2}\right)$} \\
\hline & & & & region I & region II & region III \\
\hline 1.5349 & H I & $18-4$ & & $4.3 \pm 2.0$ & $4.9 \pm 2.1$ & $1.1 \pm 0.4$ \\
\hline 1.5447 & H I & $17-4$ & & $4.2 \pm 1.5$ & $4.8 \pm 1.6$ & $1.7 \pm 0.3$ \\
\hline 1.5566 & H I & $16-4$ & & $3.7 \pm 1.2$ & $2.6 \pm 1.0$ & $1.8 \pm 0.6$ \\
\hline 1.5710 & H I & $15-4$ & & $3.3 \pm 1.1$ & $4.9 \pm 2.2$ & $2.1 \pm 0.2$ \\
\hline 1.5891 & H I & $14-4$ & & $5.4 \pm 0.6$ & $5.7 \pm 0.7$ & $2.1 \pm 0.6$ \\
\hline 1.6120 & H I & $13-4$ & & $8.4 \pm 1.9$ & $7.6 \pm 2.6$ & $3.9 \pm 1.2$ \\
\hline 1.6417 & H I & $12-4$ & & $6.8 \pm 2.1$ & $5.6 \pm 2.6$ & $4.5 \pm 0.4$ \\
\hline 1.6817 & H I & $11-4$ & & $11.6 \pm 0.8$ & $11.4 \pm 0.5$ & $7.0 \pm 0.5$ \\
\hline 1.7013 & He I & $4^{3} \mathrm{D}-3^{3} \mathrm{P}$ & & $7.3 \pm 1.6$ & $7.4 \pm 1.6$ & $3.3 \pm 0.3$ \\
\hline 1.7372 & $\mathrm{H} \mathrm{I}$ & $10-4$ & & $17.6 \pm 1.1$ & $16.8 \pm 1.0$ & $9.9 \pm 0.6$ \\
\hline 1.8185 & H I & $9-4$ & & $23.3 \pm 1.3$ & $21.8 \pm 1.8$ & $13.0 \pm 1.3$ \\
\hline 1.8696 & He I & $4^{3} \mathrm{~F}-3^{3} \mathrm{D}$ & & $7.9 \pm 0.9$ & $7.8 \pm 0.8$ & $5.7 \pm 0.7$ \\
\hline 1.8762 & H I & $4-3$ & & $183.9 \pm 1.0$ & $147.8 \pm 1.1$ & $74.8 \pm 0.7$ \\
\hline 1.9457 & H I & $8-4$ & & $31.7 \pm 1.0$ & $32.1 \pm 1.5$ & $19.5 \pm 0.8$ \\
\hline 2.0344 & $\mathrm{H}_{2}$ & $v=1-0 \mathrm{~S}(2)$ & 7584 & - & $0.6 \pm 0.4$ & $1.8 \pm 0.4$ \\
\hline 2.0592 & $\mathrm{He} \mathrm{I}$ & $2^{1} \mathrm{P}-2^{1} \mathrm{~S}$ & & $61.2 \pm 0.6$ & $60.8 \pm 0.6$ & $28.4 \pm 0.3$ \\
\hline 2.1132 & He I & $4^{1,3}-3^{1,3} \mathrm{P}$ & & $4.2 \pm 0.8$ & $4.0 \pm 0.3$ & $2.4 \pm 0.4$ \\
\hline 2.1224 & $\mathrm{H}_{2}$ & $v=1-0 \mathrm{~S}(1)$ & 6956 & - & $3.3 \pm 0.4$ & $8.5 \pm 0.2$ \\
\hline 2.1620 & $\mathrm{He} \mathrm{I}$ & $7^{3} \mathrm{~F}-4^{3} \mathrm{D}$ & & $3.1 \pm 0.4$ & $3.0 \pm 0.5$ & $1.5 \pm 0.4$ \\
\hline 2.1667 & H I & $7-4$ & & $99.4 \pm 0.7$ & $92.8 \pm 0.4$ & $57.3 \pm 0.3$ \\
\hline 2.2239 & $\mathrm{H}_{2}$ & $v=1-0 \mathrm{~S}(0)$ & 6471 & $0.0 \pm 0.7$ & $1.5 \pm 0.5$ & $3.2 \pm 0.6$ \\
\hline 2.2873 & [Se IV] & ${ }^{2} \mathrm{P}_{3 / 2}-{ }^{2} \mathrm{P}_{1 / 2}$ & & $1.2 \pm 0.4$ & $1.4 \pm 0.4$ & $1.5 \pm 0.5$ \\
\hline 2.4073 & $\mathrm{H}_{2}$ & $v=1-0 \mathrm{Q}(1)$ & 6149 & - & $7.2 \pm 1.0$ & $15.7 \pm 0.5$ \\
\hline 2.4141 & $\mathrm{H}_{2}$ & $v=1-0 \mathrm{Q}(2)$ & 6471 & - & - & $4.8 \pm 0.4$ \\
\hline 2.4244 & $\mathrm{H}_{2}$ & $v=1-0 \mathrm{Q}(3)$ & 6956 & - & $4.1 \pm 1.2$ & $6.8 \pm 0.7$ \\
\hline
\end{tabular}

Notes. Lines detected in the averaged spectra of three selected regions. The flux of each line is averaged over each region. ${ }^{(a)}$ Excitation energy adopted from Dabrowski (1984).

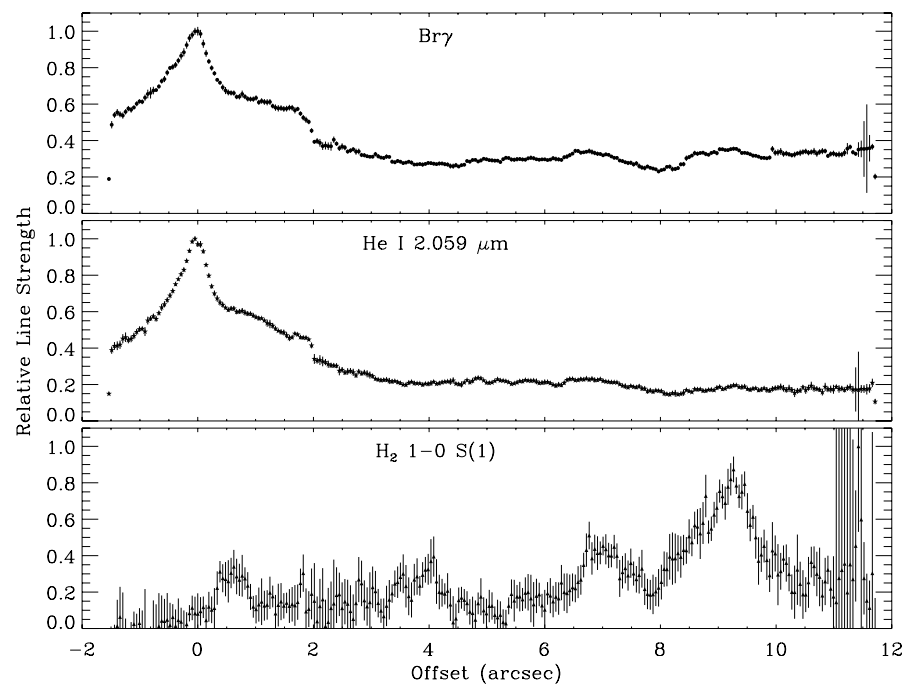

Fig. 7. Line intensity variations of three representative lines $-\mathrm{Br} \gamma$, $2.059 \mu \mathrm{m} \mathrm{He}$, and $\mathrm{H}_{2} 1-0 \mathrm{~S}(1)-$ along the strip line, which is perpendicular to the IF of M $17 \mathrm{SW}$ (outlined in Fig. 5). The zero-point is defined at 18: 20: 25.136, -16:11: 34.21 (J2000), at the maximum strength of the IF; negative offsets point toward the H II region, while positive offsets point toward the PDR.

excitation by far-ultraviolet (FUV) photons and thermal excitation in shock fronts. Practically, near-IR $\mathrm{H}_{2}$ fluorescent spectra have been observed for a variety of classical PDRs such as those associated with NGC 2023, Orion Bar, and the northern bar in M 17 (Gatley et al. 1987; Hayashi et al. 1985; Tanaka et al. 1989). Meanwhile, a variety of $\mathrm{H}_{2}$ spectra have been found to be produced in the shock fronts associated with the jets/outflows found in PDRs (e.g., Nürnberger et al. 2007; Martín-Hernández et al. 2008; Bik et al. 2010). Although these two mechanisms both act occasionally for $\mathrm{H}_{2}$ emission found in PDRs, they can be distinguished since the corresponding $\mathrm{H}_{2}$ line ratios are different.

A common way of characterizing the $\mathrm{H}_{2}$ emission is to evaluate the gas temperature in the framework of a ro-vibrational diagram, which is a plot of the observed column density against the energy of the upper level. The column density, $N_{j}$, of the upper level of a given transition can be calculated from the measured line intensity, $I$, of the corresponding $\mathrm{H}_{2}$ line via the following formula:

$N_{j}=\frac{4 \pi \lambda_{j} I}{A_{j} h c}$,

where $\lambda_{j}$ (the rest wavelength) and $A_{j}$ (the Einstein A-coefficient) are taken from Turner et al. (1977). If collisional de-excitation plays a dominant role, the $\mathrm{H}_{2}$ molecule will be in LTE and the energy level obeys the Boltzmann distribution. In this type of distribution, the relative column densities of any two excitation levels can be expressed in terms of excitation temperature $T_{\text {ex }}$

$\frac{N_{i}}{N_{j}}=\frac{g_{i}}{g_{j}} \exp \left[\frac{-\left(E_{i}-E_{j}\right)}{k T_{\mathrm{ex}}}\right]$,

where $g_{j}$ is the degeneracy, $E_{j}$ is the excitation energy taken from Dabrowski (1984), and $k$ is the Boltzmann constant. If the gas is thermalized at a single temperature, the plot of the logarithm of $N_{j}$ to $g_{j}$ ratio against the level energy will reveal a 


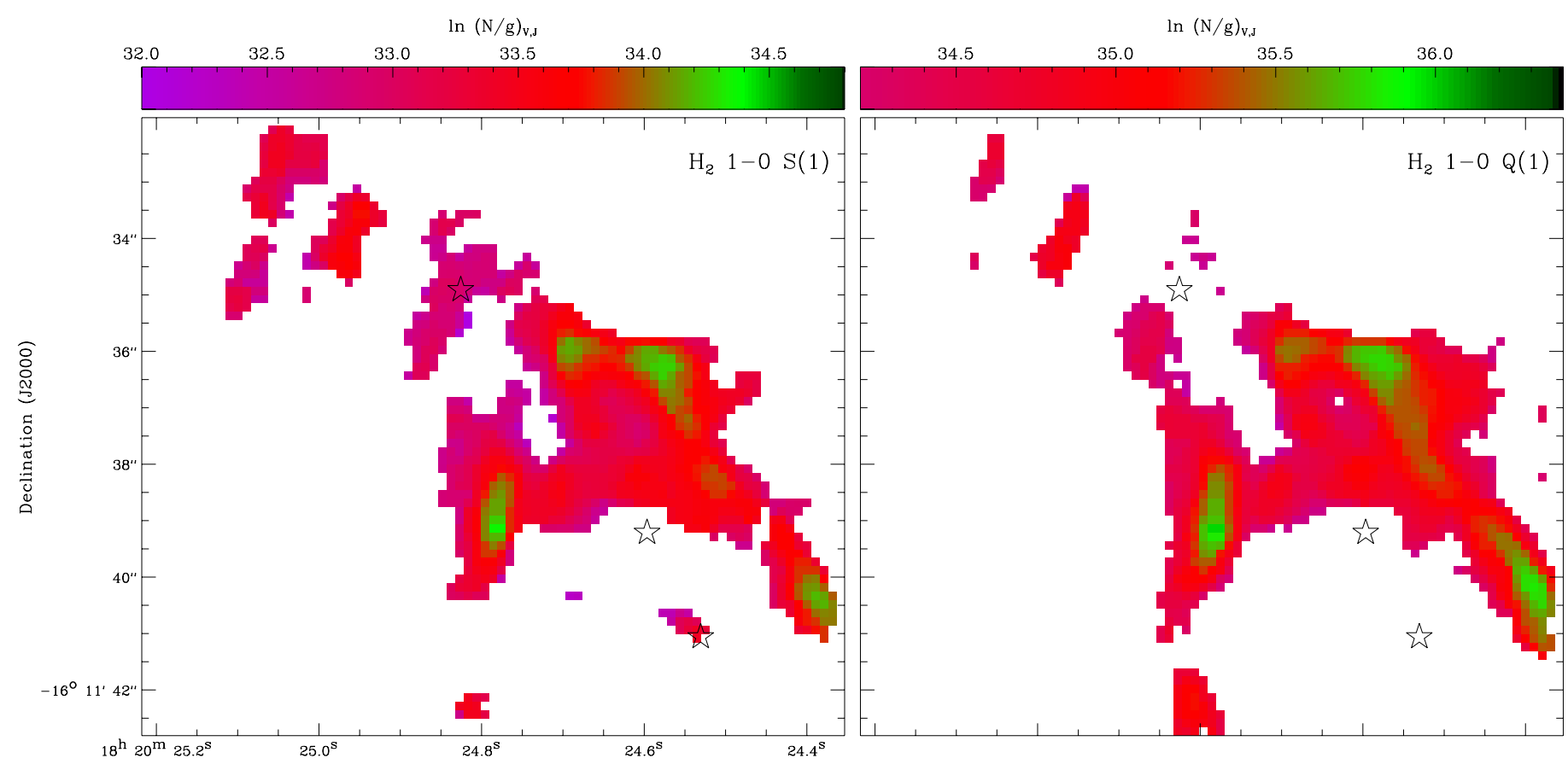

Right Ascension (J2000)

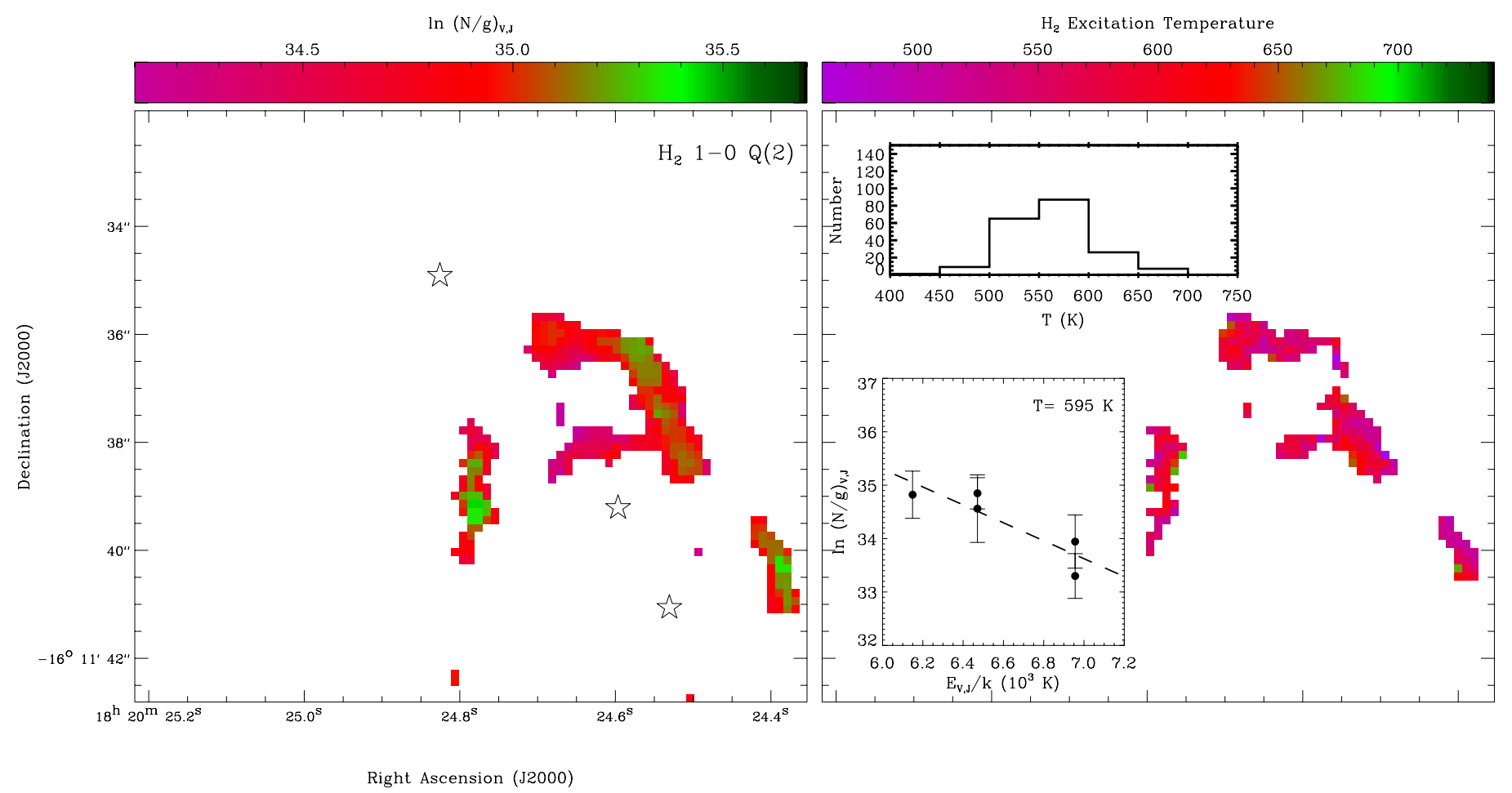

Fig. 8. Column densities of $\mathrm{H}_{2} 1-0 \mathrm{~S}(1), 1-0 \mathrm{Q}(1)$ and $1-0 \mathrm{Q}(2)$ (quadrants I to III) as well as the $\mathrm{H}_{2}$ excitation temperature map (quadrant IV). In quadrants I to III, IRS5A,C and UC1's positions are marked with asterisks. In quadrant IV, the ro-vibrational diagram based on the $\mathrm{H}_{2}$ lines $\left(\mathrm{H}_{2} 1-0 \mathrm{~S}(1), 1-0 \mathrm{~S}(0), 1-0 \mathrm{Q}(1), 1-0 \mathrm{Q}(2)\right.$, and 1-0 Q(3)) averaged over all collected pixels is plotted, as well as the histogram density of $\mathrm{H}_{2}$ excitation temperature.

straight line, whose slope provides the reciprocal of the excitation temperature.

We implied this method to evaluate the excitation temperature for the detected $\mathrm{H}_{2}$ emission lines, assuming the same dust attenuation for all lines. Figure 8 shows the extinctionuncorrected column densities of $\mathrm{H}_{2} 1-0 \mathrm{~S}(1), \mathrm{H}_{2} 1-0 \mathrm{Q}(1)$, and $\mathrm{H}_{2}$ 1-0Q(2). We excluded the $\mathrm{H}_{2}$ 1-0 S(2) line in plotting the ro-vibrational diagram because this line is too weak to be significantly detected in most FOV. Other than $\mathrm{H}_{2} 1-0 \mathrm{~S}(1)$ and $1-0 \mathrm{Q}(1)$, the remaining three $\mathrm{H}_{2}$ lines are weak, although stronger than 1-0 S(2). To increase the $\mathrm{S} / \mathrm{Ns}$ for these weak $\mathrm{H}_{2}$ lines, we degraded the angular resolution of the SINFONI data by rebinning the data with $3 \times 3$ array. The resulting $\mathrm{S} / \mathrm{Ns}$ have increased by a factor of $\sim 3$. Note that rebinning is applied merely 
when the $\mathrm{H}_{2}$ emission lines are analyzed. Thus, only Figs. 8 and 10 are plotted in the rebinned pixel scale. For $\mathrm{H}_{2} 1-0 \mathrm{~S}(1)$ and $1-0 \mathrm{Q}(1)$, a threshold of $S / N \geqslant 3$ is used; for the remaining three $\mathrm{H}_{2}$ lines, $S / N \geqslant 2$ is applied. The lower-right panel in Fig. 8 presents the map of $T_{\mathrm{ex}}$ for $\mathrm{H}_{2}$ emission, the histogram of excitation temperatures, as well as the ro-vibirational diagram based on the column densities averaged throughout the collected pixels. The ro-vibrational diagram shows that all five $\mathrm{H}_{2}$ lines lie along a straight line whose slope corresponds to an excitation temperature $\approx 600 \mathrm{~K}$. The determined $T_{\mathrm{K}}$ for each rebinned pixel might have large errors because the adopted $\mathrm{H}_{2}$ lines only cover a very narrow range of excitation temperature $(6100-7000 \mathrm{~K})$. Nevertheless, the statistical $T_{\text {ex }}$ of the $\mathrm{H}_{2}$ gas is more meaningful than a specific value for each rebinned pxiel. With the histogram of $T_{\mathrm{ex}}$ for all rebinned pixels, we found a peak around $575 \mathrm{~K}$. A single excitation temperature for the $\mathrm{H}_{2}$ emission indicates that the energy levels are thermally excited. Other discriminators for the two excitation mechanisms of $\mathrm{H}_{2}$ emission, such as $\mathrm{H}_{2} 1-0 \mathrm{~S}(1) / 2-1 \mathrm{~S}(1)$ ratio and $\mathrm{H}_{2}$ ortho-to-para ratio, both indicate thermal excitation.

For fluorescence emission, each vibrational level has $N_{j} / g_{j}$ lying along a separate "branch", and the rotational population within each level can be approximated by a thermal distribution. If there are transitions from several levels, a curved line in the ro-vibrational diagram would therefore provide evidence for non-LTE process (Martín-Hernández et al. 2008). The $\mathrm{H}_{2}$ lines at higher levels, e.g., $v=3-2$, can be useful to distinguish between the two mechanisms. In the case of the M 17 UC1-IRS5 region, the merely available $v=1-0$ transitions cannot be used to discriminate between shock and fluorescent excitation, because they will lie along a straight line in the ro-vibrational diagram for both mechanisms if uncertainties are considered. Moreover, in dense PDRs the lower $\mathrm{H}_{2}$ levels will be thermalized as in shock fronts. This degeneracy between dense PDRs and shock fronts prevents us from precisely characterizing the $\mathrm{H}_{2}$ emission from the ro-vibrational diagram. This approach is more meaningful for the regions with moderate/strong $\mathrm{H}_{2}$ emission at all transitions. Indeed, we would be curious to know whether weak $\mathrm{H}_{2}$ emission areas possess the same excitation mechanism as those with the stronger $\mathrm{H}_{2}$ emission areas.

In an $\mathrm{H}$ II region, another attempt to qualitatively characterize the $\mathrm{H}_{2}$ emission comes from the comparison of molecular and atomic hydrogen emission. The molecular-to-atomic line ratio, particularly the ratio $\mathrm{H}_{2} 1-0 \mathrm{~S}(1) / \mathrm{Br} \gamma$, is $\leqslant 1$ in active galaxies (starburst, Seyfert, and ultraluminous infrared galaxies) and star-forming regions (Hatch et al. 2005, and reference therein). Conversely, this ratio is $\geqslant 1$ in outflow regions (Hatch et al. 2005; Martín-Hernández et al. 2008). All regions showing $\mathrm{H}_{2}$ 1-0 S(1) emission yield $\mathrm{H}_{2} 1-0 \mathrm{~S}(1) / \mathrm{Br} \gamma \ll 1$. The extremely strong hydrogen atomic emission observed here indicates very intense incident stellar radiation field, which makes the FUV pumping the most likely excitation mechanism for the molecular hydrogen emission observed in our study. Further indirect evidence also points out that FUV fluorescence is the most plausible excitation mechanism. For instance, $\mathrm{H}_{2} 1-0 \mathrm{~S}(1)$ and $\mathrm{H}_{2} \quad 1-0 \mathrm{Q}(1)$ emission, seen in the top two panels in Fig. 8, shows some filament structures parallel to the IF. This $\mathrm{H}_{2}$ emission can not be characterized by the aforementioned approaches, since only the emission of 1-0 S(1) and 1-0 Q(1) are available. One may argue, however, that the $\mathrm{H}_{2}$ emission lying parallel to IF can be produced in shock waves driven by the IF. The Orion Bar PDR presents an identical case, in which the $\mathrm{H}_{2}$ emission appears behind the region, with a ratio of $\mathrm{H}_{2} 1-0 \mathrm{~S}(1) / 2-1 \mathrm{~S}(1) \sim 3$ resembling shock excitation (Hayashi et al. 1985). Nevertheless,

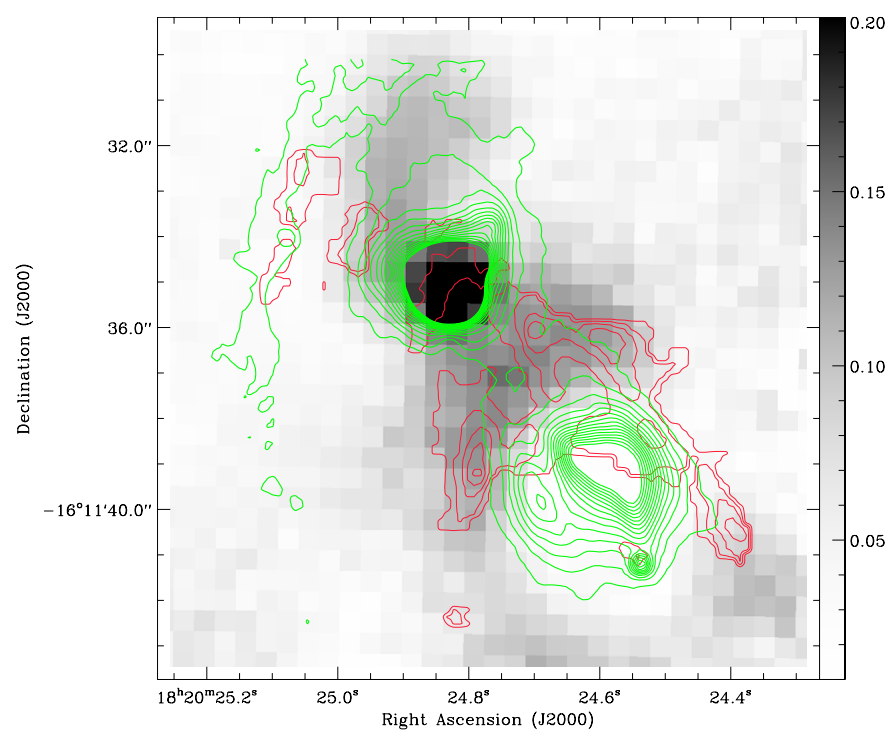

Fig. 9. $\mathrm{H}_{2} 1-0 \mathrm{~S}(1)$ line flux (red contours) overlaid on the IRSF/ $K$ polarization degree image (gray scale; $\mathrm{CZ}+12$ ). $\mathrm{H}_{2} 1-0 \mathrm{~S}(1)$ contour levels start from $2 \times 10^{-15} \mathrm{erg} \mathrm{cm}^{-2} \mathrm{~s}^{-1} \operatorname{arcsec}^{-2}$ with four intervals, each of $2 \times 10^{-15} \mathrm{erg} \mathrm{cm}^{-2} \mathrm{~s}^{-1} \operatorname{arcsec}^{-2}$. Green contours correspond to the VISIR $11.85 \mu \mathrm{m}$ emission.

Burton et al. (1990) proposed a high-density PDR model to explain the observed line ratio because the typical shock speed, $\leqslant 3 \mathrm{~km} \mathrm{~s}^{-1}$, of an expanding $\mathrm{H}$ II region driving into molecular gas is too low to significantly excite low- $v \mathrm{H}_{2}$ transitions. Considering the common properties between M17 SW and Orion Bar, we tend to neglect shock excitation for the IF-parallel $\mathrm{H}_{2}$ emission. Complementally, in Fig. 9 the IRN illuminated by IRS5A and UC1 (discussed in Sect. 4.1.2) spatially coincides with $\mathrm{H}_{2}$ emission. $\mathrm{H}_{2}$ fluorescent emission has been found for some large-scale IRN associated with star-forming regions such as NGC 2023 and Orion Bar (Gatley et al. 1987; Burton et al. 1990). Although the IRN here are associated with luminous IR stars, we speculate that this coincidence is an indirect evidence in favor of FUV fluorescence excitation.

In Fig. 10 we show the $\mathrm{H}_{2} 1-0 \mathrm{Q}(1) / 1-0 \mathrm{~S}(1)$ ratio map, which covers most of the $\mathrm{H}_{2}$ emitting regions. The $\mathrm{H}_{2}$ 1-0 Q (1)/1-0 S(1) ratio intrinsically varies with the physical conditions of $\mathrm{H}_{2}$ gas. Luhman et al. (1998) modeled $\mathrm{H}_{2}$ line ratios detected in Orion Bar and Orion $\mathrm{S}$, including $\mathrm{H}_{2}$ 1-0 Q(1)/1-0 S(1) ratio for several set of PDR models and thermal excitation by shock front as well. From their modeling, we find distinct $\mathrm{H}_{2} 1-0 \mathrm{Q}(1) / 1-0 \mathrm{~S}(1)$ ratios between PDR models and shocked thermal excitation (see Table 1 in Luhman et al. 1998). The $\mathrm{H}_{2} 1-0 \mathrm{Q}(1) / 1-0 \mathrm{~S}(1)$ ratios of the $\mathrm{H}_{2}$ emission in M17 SW are mostly in the range 1.0-3.0. The bulk of the $\mathrm{H}_{2}$ emission regions have a $\mathrm{H}_{2} 1-0 \mathrm{Q}(1) / 1-0 \mathrm{~S}(1)$ ratio $\sim 1.8$, which is between the ratios of two PDR models of $n_{\mathrm{H}}=$ $10^{6} \mathrm{~cm}^{-3}, G_{0}=10^{4}, T_{0}=500 \mathrm{~K}$, and $n_{\mathrm{H}}=10^{6} \mathrm{~cm}^{-3}, G_{0}=10^{5}$, $T_{0}=1000 \mathrm{~K}$, respectively. In contrast, the $\mathrm{H}_{2} 1-0 \mathrm{Q}(1) / 1-0 \mathrm{~S}(1)$ ratio predicted by the shocked thermal model is 0.7 , much lower than the ratios observed here. The extinction-corrected $\mathrm{H}_{2}$ 1-0 Q(1)/1-0 S(1) ratio will be smaller than that observed, however, the decrease caused by dereddenning is not big enough to lower this ratio to a thermal value.

We note quite uniform distributions of line ratios, such as $\mathrm{H}_{2} \quad 1-0 \mathrm{~S}(1) / 2-1 \mathrm{~S}(1), \quad \mathrm{H}_{2} \quad 1-0 \mathrm{~S}(1) / \mathrm{Br} \gamma$, and $\mathrm{H}_{2}$ 1-0Q(1)/1-0S(1), across all $\mathrm{H}_{2}$ emitting areas in the M 17 UC1 - IRS5 region, which indicates a uniform mechanism 


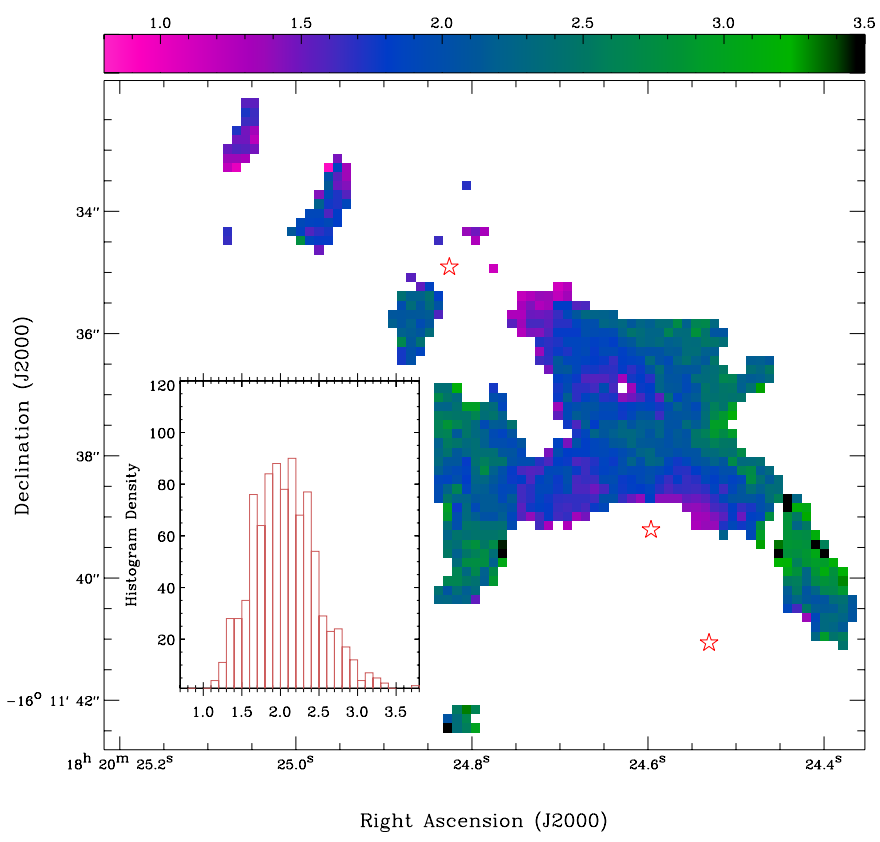

Fig. 10. Map of $\mathrm{H}_{2} 1-0 \mathrm{Q}(1) / 1-0 \mathrm{~S}(1)$ ratio and corresponding histogram density at lower left corner. The three asterisks are as same as in Fig. 8.

for the entire $\mathrm{H}_{2}$ emission. All the above properties of $\mathrm{H}_{2}$ emission can be well explained in the scenario that the $\mathrm{H}_{2}$ molecules inside M $17 \mathrm{SW}$ are initially pumped to vibrational states by FUV fluorescence, and then are de-excitated from high- $v$ levels to low- $v$ levels because of the high-frequency collision inside the high-density PDR. Because of this process, $\mathrm{H}_{2}$ emission is mostly in the form of low- $v$ vibrational states and pure rotational states. Besides the $v=1-0 \mathrm{H}_{2}$ emission lines reported here, Sheffer \& Wolfire (2013) reported mid-IR pure-rotation $\mathrm{H}_{2}$ emission at $v=0-0$ states toward M $17 \mathrm{SW}$.

\subsection{SINFONI near-IR spectroscopy of point sources}

The SINFONI $H+K$ spectra of the YSOs in M 17 UC1 - IRS5 region are crucial to understanding their fundamental properties, such as effective temperature and luminosity class, via analyzing the characteristic spectral features (see Fig. 11). Objects located inside an HII region or a PDR require special care because of contamination by nebular emission. The nebular contamination is evaluated in an annulus closely encircling the stellar contours. The spectra of IRS 5A and B273A have sufficient S/N to maintain a reliable classification, while the other sources are too faint.

The most prominent lines in Fig. 11 are the hydrogen Brackett absorption lines seen in IRS5A, the CO 2-0 bandhead absorption for $\mathrm{B} 273 \mathrm{~A}$, and the $\mathrm{HI} \mathrm{Pa} \alpha$ and $\mathrm{Br} \gamma$ emission for $\mathrm{UC1}$. The H I emission lines of UC1 are reminiscent of the common emission features of YSOs, which are due to the accretion flows falling onto the YSOs. However, we note from Fig. 5 that the emission lines mostly form in the two reflection lobes of $\mathrm{UC1}$, and a very small part comes directly from UC1, which is mostly obscured by its edge-on disk. The H I emission lines seen in UC1's spectrum arise most likely in the associated HCHII region. We suggest the shape of H I emission region oriented perpendicularly to the edge-on circumstellar disk of UC1 traces the relevant HCHII region expanding preferentially along the polar direction because of the lower density gas in polar regions.
The normalized spectra of IRS 5A and 273A are shown in Figs. 12 and 13, respectively. IRS5A's spectrum displays strong hydrogen Brackett absorption lines. The sole characteristic line in the $K$-band, $\mathrm{Br} \gamma$, indicates a temperature class later than B3, since its effective temperature is not high enough for the growth of the $2.113 \mu \mathrm{m}$ He I line, which appears for earlyB stars (Hanson et al. 1996). The Br $\gamma$ equivalent width (EW) is quantified to disentangle the ambiguity between mid-B (kB4B7) and late-B/early-A(kB8-A3); the latter shows $\mathrm{Br} \gamma \mathrm{EW}$ greater than $8 \AA$ while the former shows Br $\gamma$ EW between $4 \AA$ and $8 \AA$ (Hanson et al. 1996). The Br $\gamma$ EW of IRS5A is measured to be $4.8 \AA$, constraining its temperature class at kB4-B7. How does the $K$-band temperature class link to the optical spectral class? In the study by Hanson et al. (1996), six reference stars with known optical spectral type are assigned to a $K$-band spectral type of kB4-B7. Four out of the six stars have optical spectral classes of B3-B6, with three dwarfs and one giant. The remaining two are optically classified late-B/early-A supergiants. Hanson et al. (1996) stress that the spectral types solely based on $K$-band spectrum are not sensitive to surface gravity and show ambiguity between mid-B stars and late-B/early-A supergiants.

The $H$-band spectrum provides more spectral type indicators. The presence of strong Br11 line and weak $1.701 \mu \mathrm{m} \mathrm{He}$ I line $(E W<0.2 \AA)$ both point out a spectral class later than early-B (Hanson et al. 1998). Moreover, the Br11 EW of IRS5A is determined as $4.1 \AA$, suggesting mid-B spectral class. With the known spectral type range of IRS5A, it is possible to distinguish dwarfs and supergiants based on the $\mathrm{Br} 11 / 1.701 \mu \mathrm{mHe} \mathrm{I}$ ratio. Hanson et al. (1998) find for early/mid-B stars that this ratio is consistently larger for dwarfs than for supergiants of the same spectral class; e.g., this ratio is about 3.0 in average for B4-B7 supergiants. In the case of IRS5A, the large ratio of $\mathrm{Br} 11$ to $1.701 \mu \mathrm{m} \mathrm{He} \mathrm{I} \mathrm{(} \gtrsim 20)$ indicates a luminosity class close to dwarf.

Some He I lines (e.g., $1.701 \mu \mathrm{m}, 2.113 \mu \mathrm{m}$ ) are crucial diagnostic lines to separate early-B stars from later type stars (e.g., Hanson et al. 2005; Bik et al. 2005). Moreover, the difficulty in distinguishing dwarfs and giants solely based on near-IR spectral features also prevent us from better constraining the luminosity class of IRS5A. According to the $H+K$ spectral characteristics, we suggest a spectral type of B3-B7 V/III for IRS5A.

B273A's $H$-band spectrum does not show any significant characteristic line. Its $K$-band spectrum only shows $2.3 \mu \mathrm{m}$ CO 2-0 bandhead absorption. The CO bandhead absorption longward of $2.29 \mu \mathrm{m}$ is typical of late-type stars (e.g., Wallace \& Hinkle 1997), whose outer atmospheric layers have the proper temperatures $(\sim 1000-3000 \mathrm{~K})$ to produce such features. Moreover, low-mass Class II/III YSOs (temperatures identical to those of late-type stars) might also show CO bandhead in absorption despite the circumstellar material because the circumstellar veiling is less than that of Class I YSOs and consequently overwhelmed by the photospheric feature (Casali \& Eiroa 1996).

The CO bandhead absorption is found to be tightly related with the effective temperature and luminosity class for giants and supergiants. The EW of CO bandhead increases linearly with temperature declining for giants and supergiants, respectively, and the latter has higher EW than the former of the same effective temperature (e.g., González-Fernández \& Negueruela 2012). The CO bandhead of B273A coincides in depth and width with that of the reference star, a G4 giant (see Fig. 13). Indeed, a supergiant needs to have earlier temperature class to match the EW of a giant's CO bandhead. We measured the EW 
A\&A 578, A82 (2015)

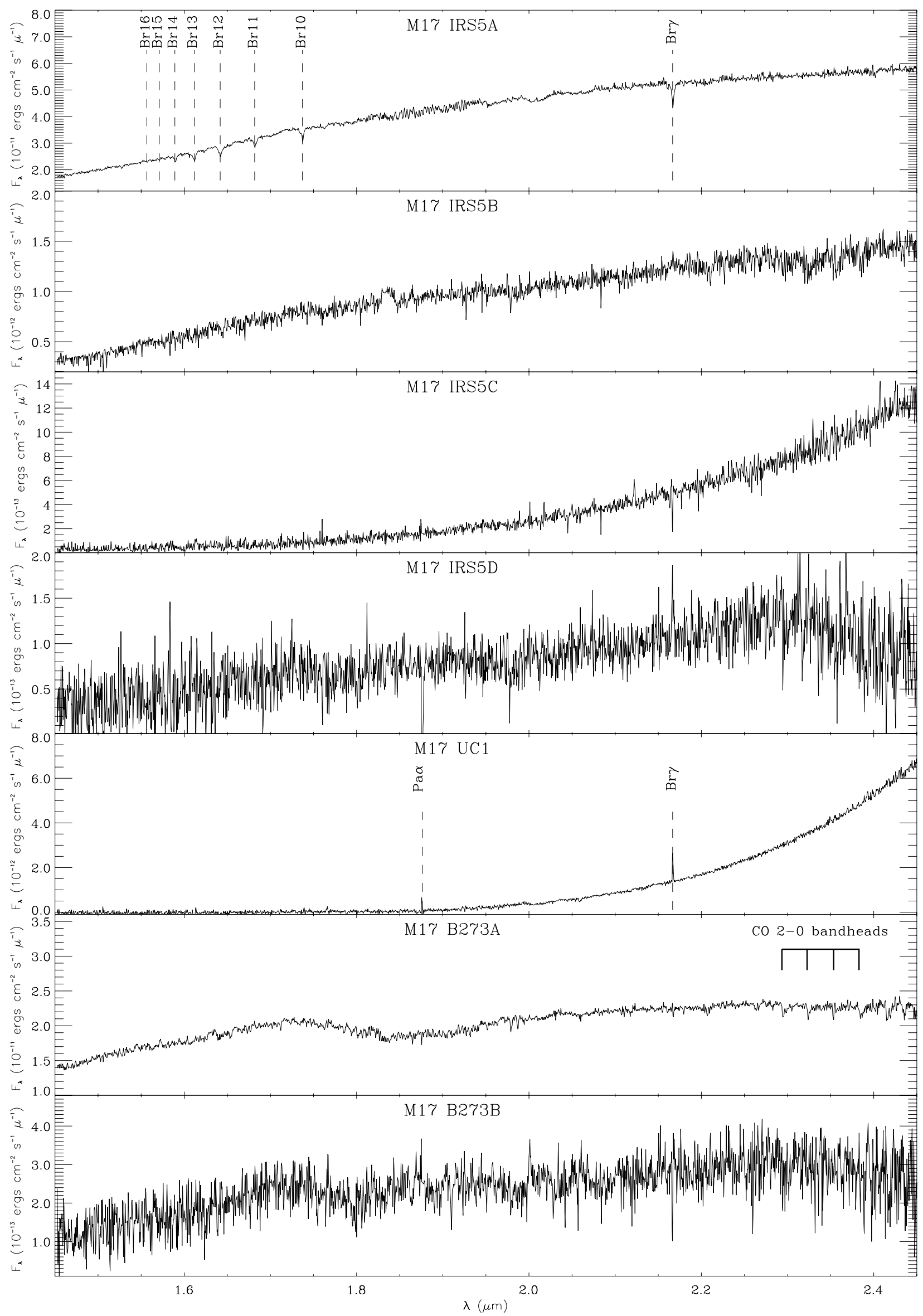

Fig. 11. Flux-calibrated SINFONI $H+K$ spectra of the point sources in M 17 UC1-IRS5 region. All natural spectral features are marked, e.g., $\mathrm{HI}$ emission/absorption lines and CO 2-0 bandheads in absorption. 


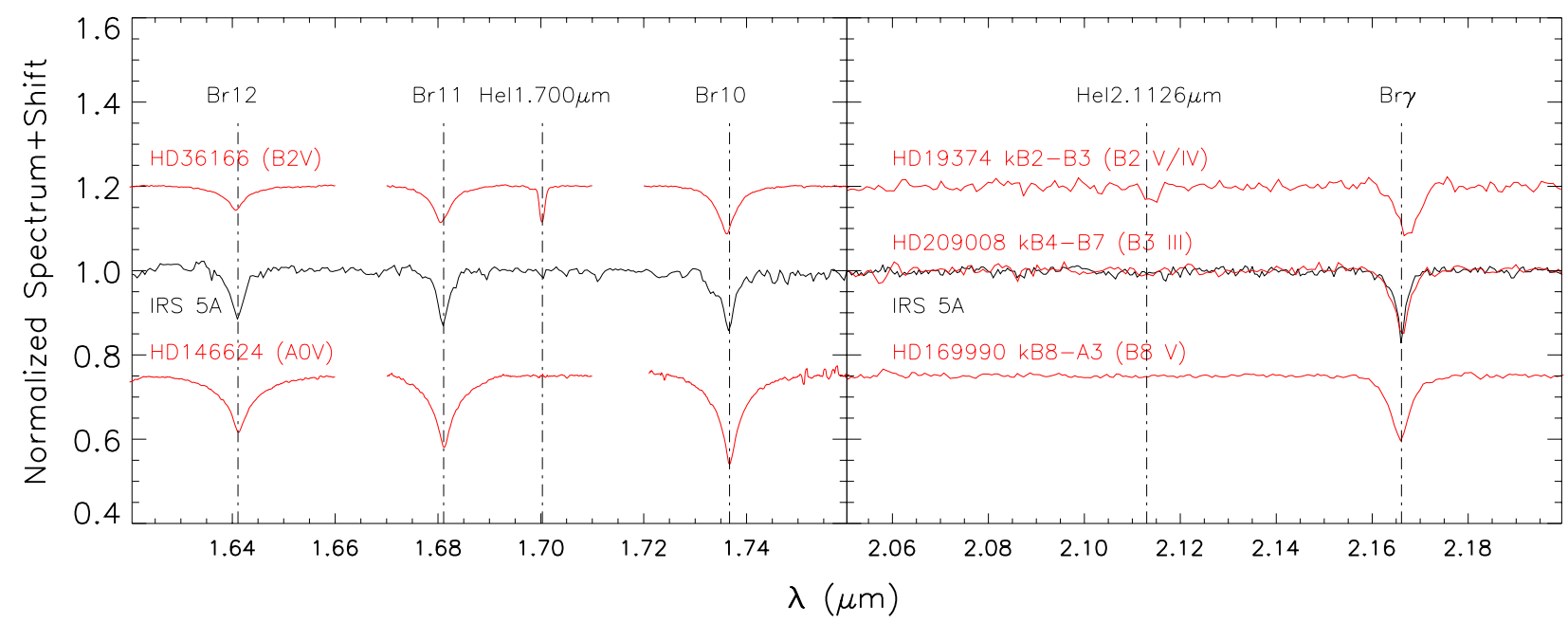

Fig. 12. Normalized SINFONI $H+K$ spectrum of IRS5A (black curve). For comparison, the spectra of several reference stars are displayed; they are adopted from the literature as follows: B2 V (HD 36166), A0 V (HD 146624) from Hanson et al. (2005); B2 V/IV (HD 19374), B3 III (HD 209008), B8 V (HD 169990) from Hanson et al. (1996). All reference spectra are converted to a resolution identical to SINFONI's resolving power.

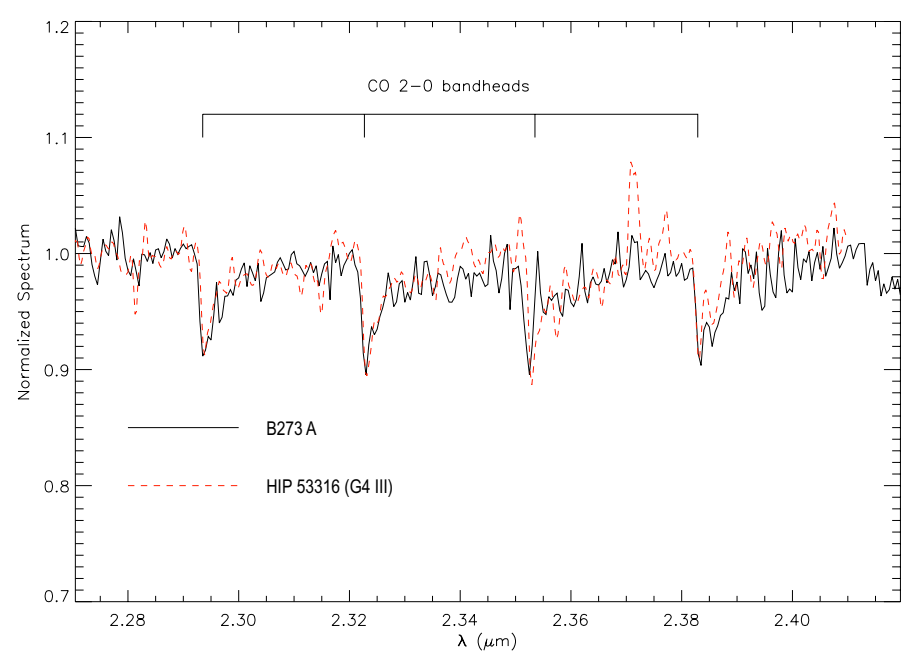

Fig. 13. Normalized SINFONI spectrum of B273A (black) in the wavelength range $2.27-2.42 \mu \mathrm{m}$, covering $\mathrm{CO} 2-0$ handhead absorption. For comparison, the reference spectrum of mid-G giant HIP 53316 (SpT G4 III; Wallace \& Hinkle 1997) is shown in red. The reference spectrum $(R \sim 3000)$ has been smoothed to the spectral resolution of SINFONI spectrum.

of CO bandhead for B273A to be $\approx 3.5 \AA$, which suggests a spectral type of G4/G5 for a giant and G2/G3 for a supergiant when following Fig. 2 in González-Fernández \& Negueruela (2012). Alternatively, we checked the EW of CO bandhead for the main-sequence stars catalogued by Wallace \& Hinkle (1997) and found that B273A's CO bandhead strength is between that of a G8 V (HR 4496) and a K2 V (HR 1084) star. We could not further constrain the spectral type of B273A without other characteristic lines at shorter wavelengths.

A fit example is CEN 34, a mid-G supergiant located along the LOS toward M 17, which is classified to be background with respect to M 17 with age between 50-100 Myr (Chen et al. 2013). Upon the consideration of B273A's apparent brightness at short wavelengths, the object should not be in the foreground of M 17. Thus we can rule out the possibility of an early-K dwarf that is only visible at shorter distance. On the other hand, the solution of early-G supergiant puts B273A in the backside of M 17.
However, the large amount of molecular gas along the LOS toward the M 17 UC1 - IRS5 region can almost obscure any background object. Therefore, we keep the solution of mid-G giant as the spectral type of B273A.

\subsection{Extinction and luminosity}

In order to place IRS5A and B273A in the Hertzsprung-Russel diagram (HRD), one has to determine their luminosity. In principle, the already constrained spectral type of the source would give some hints about deriving the extinction of the source because the temperature-dependent intrinsic color is reddened by a certain amount of extinction to the observed color. For instance, a general manner of determining the extinction for B stars is applying the short wavelength colors (e.g., $V-R$ vs. $I-R$ ) rather than the near-IR colors (i.e., $J-H$ vs. $H-K$ ), on consideration that the former are more sensitive to the temperatures of earlytype stars. In the cases of YSOs, the short wavelength colors are the only reliable way to derive the extinction value because the near-IR colors are usually affected by the strong near-IR excess emission, which is emitted by the hot circumstellar material.

The absence of hydrogen emission lines in the spectra of IRS5A and B273A suggests that there is no substantial accretion activity. We speculate that the $J-H$ color is not severely affected by dust thermal emission because of the absence of hot accretion tunnel flows. Following the extinction law at near-IR from Cardelli et al. (1989) and the specific value of $R_{V}=3.9$ toward M 17 from Hoffmeister et al. (2008), the $A_{V}$ values of IRS5A and B273A are estimated from their apparent $J-H$ colors on the basis of the intrinsic $J-H$ colors at certain temperatures (Pecaut \& Mamajek 2013). The reliability of the obtained extinction is tested by reddening a blackbody of the proper temperature with the same $A_{V}$ value. Note that the Cardelli et al. (1989) near-IR extinction law is invalid in the 5-20 $\mu \mathrm{m}$ range, hence the extinction law used by Robitaille et al. (2007) that works in the $1-20 \mu \mathrm{m}$ range is employed when reddening the SEDs. In the range $1-3 \mu \mathrm{m}$, the extinction law used by Robitaille et al. (2007) is equivalent to the Cardelli et al. (1989) extinction law when $R_{V}=3.1$. The reddened blackbody is shown in blue in Fig. 14. The blue blackbody curve matches the bluer part of the stellar SED for both objects. On the other hand, IRS5A displays 


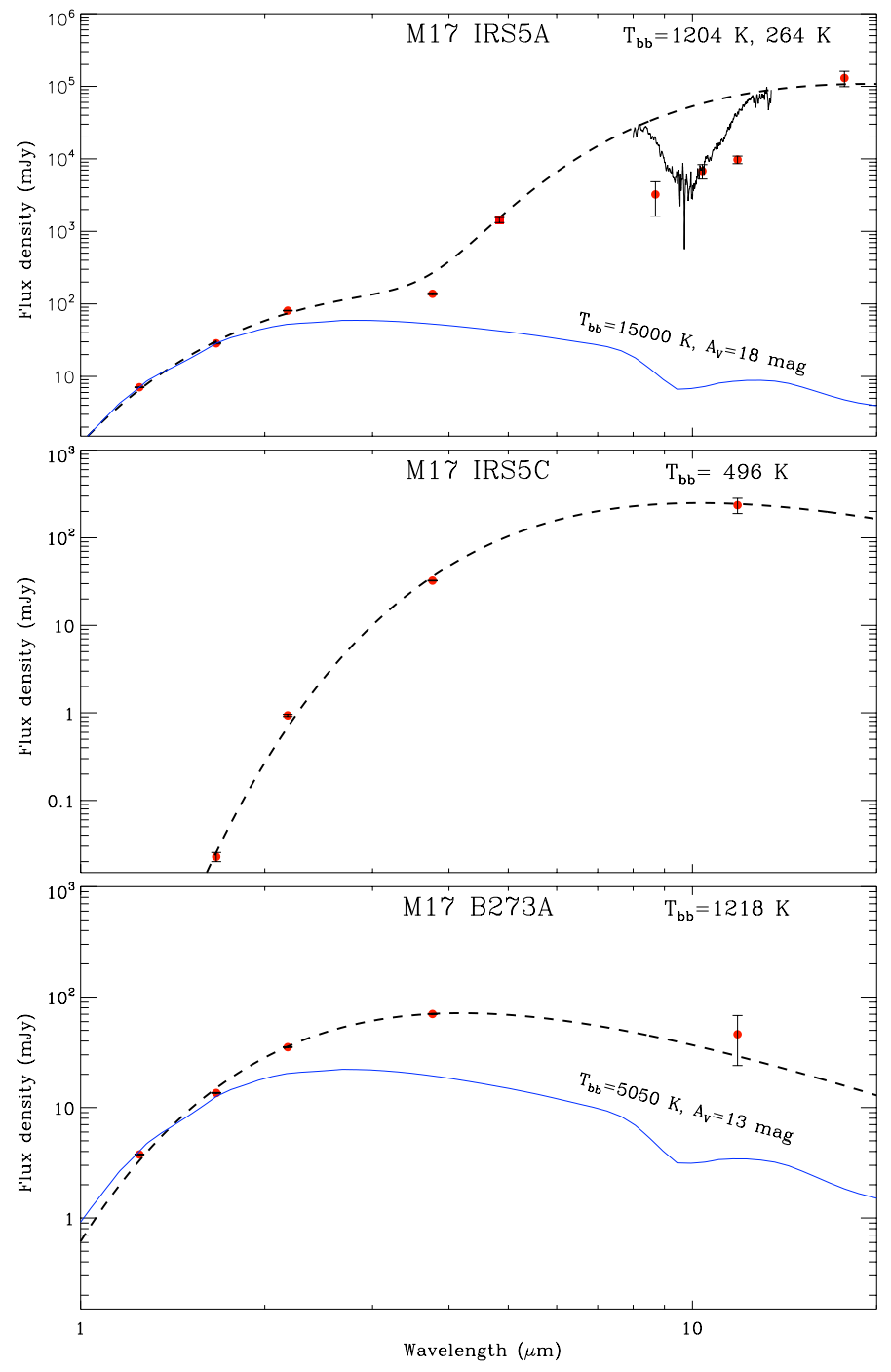

Fig. 14. SEDs constructed based on the flux measurements summarized in Tables 2 and 3 (filled circles), and the $M$-band point by ISAAC (filled square; Hoffmeister 2008). For IRS5A, the TIMMI2 spectrum at around $9.7 \mu \mathrm{m}$ is denoted by the solid curve in the top panel. The temperature of the blackbody (in blue) corresponds to the proper spectral type according to Cox (2000).

a deep $9.7 \mu \mathrm{m}$ silicate absorption feature. Using the empirical relation for silicate optical depth $A_{V}=(18 \pm 1) \tau_{9.7}$ (Whittet 2003), IRS5A's $A_{V}$ is estimated as $\sim 41 \mathrm{mag}$ from $\tau_{9.7} \sim 2.3$. Note that $A_{V}$ of IRS5A is even higher than that ( $\left.40 \mathrm{mag}\right)$ of UC1 $(\mathrm{N}+07)$, while the latter is redder than the former. This contradiction even could not be explained by the difference in spectral type between them. Alternatively, if we adopt $A_{V} / \tau_{9.7}=9-19$ (Roche $\&$ Aitken 1985), $A_{V}$ of IRS5A is hence between 21-44 mag. The $A_{V}$ based on $\tau_{9.7}$ has a large error, which originates from the various $A_{V} / \tau_{9.7}$ ratios. The $A_{V}$ value of IRS5A calculated from its $J-H$ color agrees with the upper limit from optical data. We adopt the $A_{V}$ values of IRS5A and B273A derived by their $J-H$ colors (see Table 5).

The visual extinction $A_{V}$ evaluated above is then converted to $A_{J}$ at $J$-band following the relation $A_{J} \approx 0.27 A_{V}$ when $R_{V}=3.9$. Further, the $J$-band absolute magnitude $\left(M_{J}\right)$ can be estimated for both IRS5A and B273A through distance modulus. For IRS5A, the value of $M_{J}$ is -3 mag. Referring to Pecaut $\&$ Mamajek (2013), the temperature of IRS5A $(\sim 15000 \mathrm{~K})$ corresponds to a $J$-band bolometric correction factor $\left(\mathrm{BC}_{J}\right)$ of
Table 5. Infrared luminosity of the YSOs in the M17 UC1-IRS5 region.

\begin{tabular}{lccc}
\hline \hline Object & $\begin{array}{c}A_{V} \\
(\mathrm{mag})\end{array}$ & $\begin{array}{c}L_{\mathrm{IR}}{ }^{a} \\
\left(L_{\odot}\right)\end{array}$ & $\begin{array}{c}L_{\mathrm{bol}} \\
\left(L_{\odot}\right)\end{array}$ \\
\hline M 17 B273A & 13 & $10(44)$ & 71 \\
M 17 UC1 & 40 & $2.5 \times 10^{3}\left(1.1 \times 10^{4}\right)$ & $3.0 \times 10^{4}$ \\
M 17 IRS5A & 18 & $2.0 \times 10^{3}\left(3.0 \times 10^{3}\right)$ & $8.6 \times 10^{3}$ \\
M 17 IRS5C & - & 13 & - \\
\hline
\end{tabular}

Notes. ${ }^{(a)}$ Values in brackets are extinction-corrected $L_{\mathrm{IR}} .{ }^{(b)}$ Values adopted from $\mathrm{N}+07$.

$-1.6 \mathrm{mag}$. Hence, the bolometric magnitude, $M_{\mathrm{bol}}$, of IRS5A is estimated to be $-4.6 \mathrm{mag}$, corresponding to a bolometric luminosity $\approx 5500 L_{\odot}$. Here the solar $M_{\text {bol }}$ is adopted as $+4.75 \mathrm{mag}$. Similarly, we have $M_{J} \approx-1.0 \mathrm{mag}$, and $\mathrm{BC}_{J} \approx 1.25$ for B273A $\left(T_{\text {eff }} \sim 5200 \mathrm{~K}\right) . M_{\text {bol }} \approx 0.25$ of B273A is equal to a bolometric luminosity of $63 L_{\odot}$.

The flux measurements from near- to mid-IR wavelengths provide an observational approach to evaluate the luminosity. With the new flux measurements in the $1-20 \mu \mathrm{m}$ range we present here, we are able to update the IR luminosity of IRS5A, and derive the IR luminosity for IRS5C and B273A. As shown in Fig. 14, we employ the simple blackbody curve to fit the infrared flux of the three objects. The significant absorption dip around $10 \mu \mathrm{m}$ in IRS5A's SED corresponds to the $9.7 \mu \mathrm{m}$ silicate absorption, which is confirmed by the TIMMI 2 spectrum. Hence the flux measurements at wavelengths around $9.7 \mu \mathrm{m}$ are not used in fitting the SED of IRS5A. However, for the SEDs of IRS5C and B273A, because the $11.85 \mu \mathrm{m}$ flux is the only available measurement in mid-IR, we still use this flux measurement in fitting. The $9.7 \mu \mathrm{m}$ silicate absorption should be taken into account when dereddening the SED. The $11.85 \mu \mathrm{m}$ flux is affected by the $9.7 \mu \mathrm{m}$ silicate absorption feature, thus the blackbody fitting overestimates the color temperatures of IRS5C and B273A.

The SED of IRS5A can be well fitted by two blackbody components with color temperatures of 1204 and $264 \mathrm{~K}$. The cooler component indicates a large amount of dust grains along the LOS of IRS5A. The color temperatures of IRS5C and B273A are $496 \mathrm{~K}$ and $1218 \mathrm{~K}$, respectively. The observed IR luminosity in the $1-20 \mu \mathrm{m}$ range, $L_{\mathrm{IR}}$, of these objects are summarized in the third column of Table 5 .

IRS5A's $L_{\mathrm{IR}}$ is very close to the values in $\mathrm{N}+01$ and $\mathrm{K}+02$, which reported values of $3000 L_{\odot}$ and $2600 L_{\odot}$ for IRS5, respectively. In their work, the aperture size is larger than the one we use, and IRS5C is not resolved in their larger beam infrared images. The smaller luminosity that we derive for IRS5A is reasonable. On the other hand, the observed $L_{\mathrm{IR}}$ of IRS5A and B273A are much lower than their bolometric luminosity $L_{\mathrm{bol}}$ derived above. If the observed flux measurements are extrapolated to longer and shorter wavelengths, and are corrected for the extinction, the $L_{\text {bol }}$ of IRS5A and B273A can be constrained observationally.

The extinction-corrected $L_{\mathrm{IR}}$ is obtained for IRS5A and B273A, using the proper extinction law mentioned above. Furthermore, the $L_{\mathrm{bol}}$ of IRS5A and B273A are derived by extrapolating the best-fitting blackbody to longer and shorter wavelengths, e.g., in the range $0.02-200 \mu \mathrm{m}$. The obtained $L_{\mathrm{bol}}$ is listed in the fourth column of Table 5.

The $L_{\text {bol }}$ of IRS5A and B273A derived from SED fitting are compared with the values obtained from the temperaturedependent bolometric correction values. For IRS5A, the former 


\section{Z. Chen et al.: VLT IR study of M 17 UC1 - IRS5 region}

value is significantly larger than the latter. This difference is partly caused by the extinction used in dereddening the SED of IRS5A. IRS5A shows evidence of circumstellar material; the extinction caused by the circumstellar material is included when dereddening the SEDs. However, the circumstellar extinction does not attenuate the $L_{\mathrm{bol}}$, but simply redistributes the SED because the circumstellar material absorbs the blue photons and re-emits at longer wavelengths if assuming an ideal blackbody. In the case of IRS5A, the circumstellar extinction might be a small portion of the total extinction, upon consideration that the foreground extinction toward the southwestern bar is suggested to be on the order of 10 mag or even higher (e.g., Pellegrini et al. 2007; Sheffer \& Wolfire 2013). We recalculated the $L_{\text {bol }}$ with a smaller $A_{V}$ value by 1 mag difference, and got smaller $L_{\mathrm{bol}}$ of $5.8 \times 10^{3} L_{\odot}$ for IRS5A, which agrees much better with the value of $5.5 \times 10^{3} L_{\odot}$. Therefore, we take the $L_{\text {bol }}$ values in Table 5 as the upper limits, and the extinction-corrected $L_{\mathrm{IR}}$ as the lower limits in the following discussions.

\section{Discussion}

\subsection{Multiplicity}

With the high angular resolution infrared images toward M 17 UC1 - IRS5 region, the companions of the luminous infrared objects (IRS5, B273) are resolved for the first time. According to the $H K L$ color-color diagram, the two components of the B273 system both show $L$-band excess emission. Thus, we suggest that B273A and B273B form a young binary system with a projected separation of about $2200 \mathrm{AU}$. Three of the five components of IRS5 show $L$-band excess emission. In light of IRS5D's position in Fig. 2, we also regard it as a member of IRS5 system taking the photometric errors into account, and suggest IRS5 might be a young quadruple system.

\subsection{Structures of the circumstellar material}

The large mid-IR excess emission of IRS5A and UC1 previously reported by $\mathrm{N}+01$ and $\mathrm{K}+02$ indicate circumstellar material for both objects. In addition, from a near-IR polarization view $(\mathrm{CZ}+12)$, the high-level polarization individually associated with IRS5A and UC1 also traces dust grains physically linked with the two objects. Specifically, the almost edge-on circumstellar disk of $\mathrm{UC} 1$ found by $\mathrm{N}+07$ produces the high-level $K_{\mathrm{s}}$-band polarization vectors parallel to the disk. The polarization pattern of the IRN illuminated by IRS5A, however, implies a very different orientation than $\mathrm{UC1}$; e.g., the inclination angle of IRS5A's IRN estimated in $\mathrm{CZ}+12$ is $\sim 50^{\circ}$ from the plane of the sky.

Figure 9 presents perfect geometric match between the $\mathrm{H}_{2}$ emission and polarized light, which explains the physical carriers of the polarized light. The $\mathrm{H}_{2}$ emission residing just beside $\mathrm{UC} 1$ is exactly aligned with the elongated feature extending to the northwest, which we suggest coincides with the dust thermal emission originated in the circumstellar disk of UC1 (see Sect. 4.2). We speculate that such $\mathrm{H}_{2}$ emission is related to the circumstellar disk of $\mathrm{UC} 1$, and actually traces the warm $\mathrm{H}_{2}$ gas in UC1's disk. The dust grains in UC1's disk scatter the incident starlight, and produce the polarized light seen at near-IR. The $\mathrm{H}_{2}$ emission filament related to UC1's disk is one of the three $\mathrm{H}_{2}$ emission filaments that are parallel to each other, and parallel to the IF. The $\mathrm{H}_{2}$ filament at the midway between the IF and UC1 is spatially coinciding with the bar-like emission feature seen in the NACO/K image (see Sect. 4.1.2 and Fig. 3).
Moreover, the area covered by this bar-like emission in turn is also occupied by the south-north mid-IR extended emission originated from UC1. This south-north extended emission seen in mid-IR and the coordinated IRN seen in near-IR polarized light together suggest a south-north outflow from UC1. The bar-like emission seems to arise at the northeastern side of this outflow because this side is facing the IF. The IF northeast of the outflow suppresses the outflowing material on the northeastern side, and consequently forms a sheet of gas with a higher temperature than the rest of the outflow. The temperature difference between the sheet at outflow's northeastern side and the rest of the outflow explains well the different morphology of the outflow seen between near-IR and mid-IR wavelengths. Moreover, the coordinated emitting $\mathrm{H}_{2}$ gas at the northeastern side of the outflow is possibly excited at a temperature of $\sim 600 \mathrm{~K}$ (see Sect. 4.3.3), while we suggest the rest of the outflow has thermal temperature around $100 \mathrm{~K}$ heated by an early-B outflow driving object (Longmore et al. 2011). The north-south outflow of UC1 is most likely the blue-shifted lobe, with the red-shifted lobe being invisible because of severe attenuation.

The coordinated emitting $\mathrm{H}_{2}$ gas surrounding IRS5A and the IRN illuminated by the same object indicates a distribution of gas and dust embracing IRS5A in the north with an arc-like shape. The circular shape of the IRN illuminated by IRS5A reminds us that the southern half might be associated with $\mathrm{H}_{2}$ gas too, although the southern half is not fully covered by the SINFONI data in its small FOV. The temperature of this material distribution is $\sim 600 \mathrm{~K}$, similar to the associated emitting $\mathrm{H}_{2}$ gas. With the high-resolution VISIR $11.85 \mu \mathrm{m}$ image, we expect a tight relation between the diffuse mid-IR emission and the IRN, as has been observed for UC1. However, the diffuse mid-IR emission is only observed at the midway between IRS5A and UC1, where merely a portion of the IRN is spatially associated (see Sect. 4.2). Indeed, this mismatch can be the result of two separate material distributions that are along the same LOS; the dust temperature responsible for the mid-IR emission of IRS5A is modeled at around $264 \mathrm{~K}$, while the dust temperature linked with the IRN and $\mathrm{H}_{2}$ emission is fitted at around $575 \mathrm{~K}$. The two lobes in the core region of IRS5A stack with the inner circle of the arc-like material along the LOS. The different morphology of IRS5A seen at near-IR and mid-IR raise the question of whether the mid-IR emission comes from the area close to IRS5A or from its envelope or outflow. The dark lane separating the two mid-IR lobes can somehow be explained by a nearly edge-on cold disk that absorbs the mid-IR emission from backside. The proposed cold disk should have impacts at nearIR bands, such as a silhouette dividing the object into two lobes as well, very similar to UC1. Nevertheless, IRS5A's near-IR images do not show any sign of a silhouette disk. The extinction of IRS5A is another strong argument against the assumption of a nearly edge-on cold disk because the circumstellar extinction is only a small portion of the total extinction of IRS5A. We propose that the mid-IR emission actually arises from the outflow whose axis is almost along the LOS of IRS5A. The two lobes seen in mid-IR correspond to the outflow walls at two sides. In view of the severe attenuation at near-IR bands and at around $10 \mu \mathrm{m}$ (Chapman et al. 2009), the $\mathrm{H}_{2}$ emission filament is the blue-shifted lobe. Meanwhile, the outflowing material traced by the mid-IR emission might be blue-shifted because of the attenuation issue too.

Figure 8 indicates that IRS5C is closely associated with $\mathrm{H}_{2} 1-0 \mathrm{~S}(1)$ emission. Indeed, IRS5C is embraced by $\mathrm{H}_{2}$ 1-0 S(1) emission, which shows an elliptical shape orientated at $\mathrm{PA} \sim 65^{\circ}$. The bulk of $\mathrm{H}_{2}$ emission is shifted northeast 


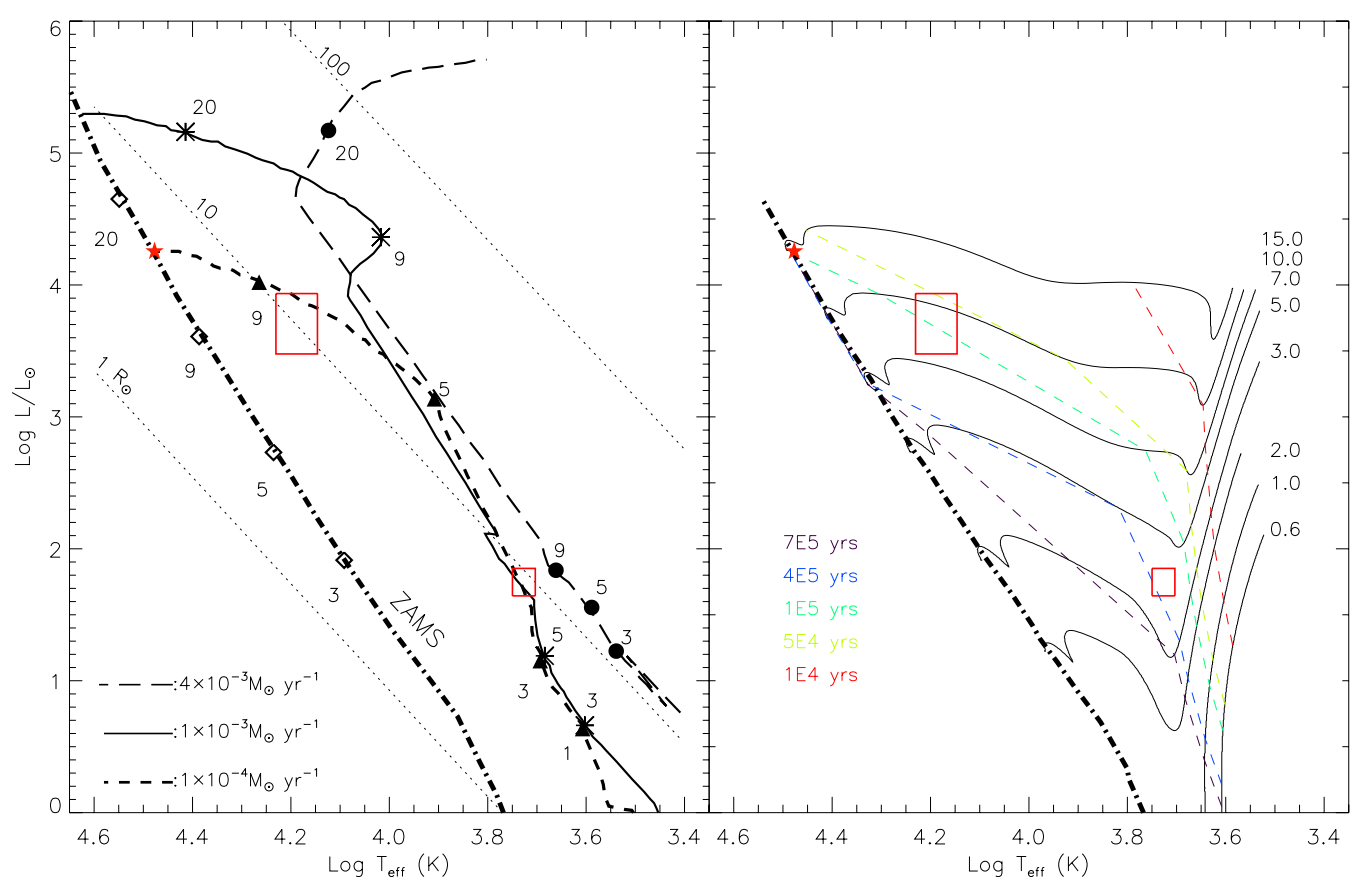

Fig. 15. Positions of the YSOs in the HRD with well-constrained temperatures and luminosities. Red rectangles outline the parameter space of IRS5A (the upper one) and B273A (the lower one), and the red asterisk at upper left marks UC1. For IRS5A and B273A, the conversion from spectral types to effective temperatures and bolometric luminosities is referred to Sect. 4.5, while UC1 is assumed to be a ZAMS B0.5 star in light of the HCHII region $(\mathrm{N}+07$ and references therein). Left panel: positions of the YSOs compared with the model of massive star formation via disk accretion $(\mathrm{H}+10)$. The symbols along the evolutionary loci at various accretion rates and the ZAMS locus denote the mass at each position. Right panel: positions of the YSOs placed against the PMS evolutionary tracks of different masses (CA+12). Isochrones of five ages are drawn as dashed lines in different colors.

to IRS5C. As described in Sect. 4.1.1, IRS5C coincides with the 22- $\mathrm{GHz} \mathrm{H}_{2} \mathrm{O}$ maser, which is located 0.'5 northwest to IRS5C. The $\mathrm{H}_{2} 1-0 \mathrm{~S}(1)$ emission associated with IRS5C resembles that found for some Herbig Ae/Be stars in their circumstellar disks, where molecular $\mathrm{H}_{2}$ gas is heated by the $\mathrm{Ae} / \mathrm{Be}$ stars themselves (Carmona et al. 2011). The strong excess emission at $L$-band (this work) and mid-IR indicates the presence of circumstellar material surrounding IRS5C. Hence it is plausible that $\mathrm{H}_{2}$ gas among the warm circumstellar material thermally emits $\mathrm{H}_{2} 1-0 \mathrm{~S}(1)$ emission detected here. However, unlike the $\mathrm{H}_{2} 1-0 \mathrm{~S}(1)$ emission detected in the circumstellar disks of HD 97048 and HD 100546, which originates at radii on the order of $\sim 10^{1}-10^{2} \mathrm{AU}$, the size of the $\mathrm{H}_{2} 1-0 \mathrm{~S}(1)$ emission associated with IRS5C is about $1600 \mathrm{AU}$ in diameter. $\mathrm{H}_{2}$ emission occurring at large distances requests massive stars other than Herbig Ae/Be stars to maintain a sufficient radiation field. Nevertheless, the other possibility is that the $\mathrm{H}_{2}$ emission is externally excited by the FUV photons from the ionizing OB stars in M 17. In light of the excitation mechanism of the $\mathrm{H}_{2}$ emission associated with other objects, the latter explanation is more plausible and reliable. To explain the location of the $\mathrm{H}_{2}$ emission, we propose a nearly edge-on flared disk with radius of $1600 \mathrm{AU}$ to approximately match the shape of $\mathrm{H}_{2}$ emission. In addition, the $22-\mathrm{GHz} \mathrm{H}_{2} \mathrm{O}$ maser resides 0.' 5 away along the axis of the circumstellar disk of IRS5C. In some cases, the $\mathrm{H}_{2} \mathrm{O}$ masers can be excited in the shock fronts where the outflowing gas from highmass YSO strongly interacts with the surrounding molecular gas (e.g., Torrelles et al. 2014). Considering the position of the $\mathrm{H}_{2} \mathrm{O}$ maser with respect to the proposed disk and the disk's size, we interpret that IRS5C is a deeply embedded high-mass protostar with a nearly edge-on flared disk. IRS5C does not show radio free-free emission at $1.3 \mathrm{~cm}$ (Johnson et al. 1998), which implies that IRS5C is still at an early stage.

\subsection{Evolutionary stages of YSOs}

With the known temperature and luminosity for IRS5A and B273A, the locations of these two objects in the HRD reveals their evolutionary stages when compared with numerical models for star formation. The model of $\mathrm{H}+10$ specifically considers the evolution of a massive protostar via disk accretion, while that of Claret (2012) models the PMS evolution purely tuned by the gravitational contraction without mass feeding, i.e., nonaccretion model.

Both models indicate that IRS5A is still experiencing PMS stage. Using the accretion model $\mathrm{H}+10$ to estimate IRS5A's mass and age is problematic because of the constant accretion rate and fixed initial condition used in $\mathrm{H}+10$. If referring to the nonaccretion PMS model, IRS5A's mass is currently about $9 M_{\odot}$ and will not change much when IRS5A is evolving toward ZAMS. And a nonaccretion $0.1 \mathrm{Myr}$ isochrone passes by IRS5A's position in the HRD. The nonaccretion model simplifies the evolution of protostars, whose accretion histories, initial radii, and thermal efficiencies can greatly alter their positions in the HRD. Nevertheless, very young age estimates $(<1 \mathrm{Myr})$ for stars hotter than $3500 \mathrm{~K}$, based on the nonaccretion model, are reliable (Hosokawa et al. 2011). Therefore, we suggest that the age estimate for IRS5A is reliable.

However, the circumstellar material encircling IRS5A, which is found to be the bipolar molecular outflow driven by itself, and the lack of accretion tracers both place in doubt that IRS5A would continue accretion to gain mass. The peculiarity of this object is that it currently does not show any tracer of accretion funnel flow, which implies either periodic accretion or feedback (radiation pressure, outward mass flow) halted accretion. Both mechanisms are thought to have impact on the final mass of high-mass protostars, hence are crucial to understanding 
the formation of high-mass protostars. Otherwise, the detection limit of the SINFONI data could also prevent us from resolving weak hydrogen emission lines (see the discussion in Sect. 5.4). Follow-up investigations for the gas kinematic in the close vicinity of IRS5A will contribute to determining the gas motion and consequently clarify the evolution trend via constraining the accretion status. These follow-up studies will require high spatial resolution and high spectral resolution observations, which can be implemented by Atacama Large Millimeter/submillimeter Array (ALMA).

B273A is located in the right-lower area of HRD. Model $\mathrm{H}+10$ interprets mass gaining for B273A, however, it seems to lack hydrogen recombination lines. The excess emission at $L$-band and mid-IR both indicate the presence of circumstellar material, e.g., a circumstellar disk. Hence, we propose phases of periodic accretion or halted accretion for B273A, while the emission line strength below the detection limit could be plausible too. In the other case, the location of B273A in the right panel of Fig. 15 suggests a mass $\sim 4 M_{\odot}$ and an age $\sim 4 \times 10^{5}$ yrs. As a similar case to IRS5A, we believe that B273A's age estimate based on the nonaccretion PMS model is reliable, and B273A is still at the first half stage of its entire evolution life toward ZAMS.

\subsection{Feedback on accretion}

One may argue that the spectra of IRS5A and B273A have a low resolution $(R \sim 1500)$ and not high enough $\mathrm{S} / \mathrm{N}$, which leads to a "fake" nondetection in case of weak $\mathrm{Br} \gamma$ emission. Based on the spectral resolution $(F W H M \approx 3$ pixels $)$ and continuum's noise level, we estimated that the upper limit of $\mathrm{Br} \gamma$ emission flux is $\sim 2.2 \times 10^{-15} \mathrm{erg} \mathrm{cm}^{-2} \mathrm{~s}^{-1}$ for IRS5A and $7.9 \times 10^{-16} \mathrm{ergs} \mathrm{cm}^{-2} \mathrm{~s}^{-1}$ for B273A; with the extinction values of these two objects shown in Table 5, the extinction-corrected $\mathrm{Br} \gamma$ line flux, $f_{\mathrm{Br} \gamma}$, is $\sim 1.3 \times 10^{-14} \mathrm{erg} \mathrm{cm}^{-2} \mathrm{~s}^{-1}$ for IRS5A and $\sim 2.8 \times 10^{-15} \mathrm{erg} \mathrm{cm}^{-2} \mathrm{~s}^{-1}$ for B273A. Further, the luminosity of $\mathrm{Br} \gamma$ emission line was computed as $L_{\mathrm{Br} \gamma}=4 \pi d^{2} f_{\mathrm{B} r}$, where $d$ is the distance of M 17. The Bry line luminosity is found to be tightly correlated with the accretion luminosity, $L_{\text {acc }}$, for a statistical sample of low-mass YSOs (e.g., Mendigutía et al. 2011; Rigliaco et al. 2012; Alcalá et al. 2014). For high-mass YSOs, the correlation between $L_{\mathrm{Br} \gamma}$ and $L_{\mathrm{acc}}$ is not well established because of the lack of a statistically meaningful sample. As an approximation, we used the $\log L_{\mathrm{Br} \gamma}-\log L_{\mathrm{acc}}$ relation reported in Alcalá et al. (2014) to estimate the $L_{\text {acc }}$, which is $\sim 2.2 L_{\odot}$ for IRS5A, and $\sim 0.38 L_{\odot}$ for B273A. From the positions of these two objects in the HRD, we estimated a radius of $\sim 10 R_{\odot}$ for both of them. Together with the masses of the two objects mentioned above, the formula

$$
\dot{M}_{\mathrm{acc}} \approx 1.25 \frac{L_{\mathrm{acc}} R_{*}}{G M_{*}}
$$

gives the upper limit of $\dot{M}_{\text {acc }}$, which is $\sim 1.1 \times 10^{-7} M_{\odot} \mathrm{yr}^{-1}$ for IRS5A, and $\sim 3.8 \times 10^{-8} M_{\odot} \mathrm{yr}^{-1}$ for B273A.

High-mass stars can still arrive on the ZAMS stage during their accretion phase. However, the enormous radiation pressure and ionizing photons will act on the accretion flow and dissipate the circumstellar material. Thus, the timescale of the accretion phase for a high-mass protostar will greatly depend on the stellar mass, which in turn is constrained by accretion rate. This timescale is far from being known for high-mass protostars. UC1's disk suggests that this B0.5 ZAMS star might still be in its accretion phase. Plausible evidence supporting accretion comes from the $\mathrm{Br} \gamma$ emission at the position of UC1's southwest lobe (Fig. 5). Nevertheless, Br $\gamma$ emission can be produced in the HCHII region associated with UC1. For an HCHII region, the dynamics and morphology of the ionized gas are determined by the ratio of the ionization radius, $R_{i}$, and the gravitational bound radius, $R_{\mathrm{b}}$, defined by $G M_{*} / 2 c_{\mathrm{s}}^{2}$, where $M_{*}$ is the stellar mass and $c_{\mathrm{s}}$ is the sound speed (Keto 2007). With $M_{*}=15 M_{\odot}$ and $c_{\mathrm{s}}=10 \mathrm{~km} \mathrm{~s}^{-1}$ (at the temperature of $10^{4} \mathrm{~K}$ for ionized gas), $R_{\mathrm{b}}$ is $66 \mathrm{AU}$. Since the $\mathrm{Br} \gamma$ emission must come from the ionized gas of the HCHII region, the $\mathrm{Br} \gamma$ line map in Fig. 5 shows the morphology of the HCHII region around UC1. The distance from the obscured centroid of UC1 to the southwestern $\mathrm{Br} \gamma$ emission knot is taken as the value of $R_{i}$, which is $\sim 0$.' 25 , or $\sim 500 \mathrm{AU}$. Thus, for $\mathrm{UC} 1, R_{i} \gg R_{\mathrm{b}}$, corresponding to a stage of HCHII region that the ionized gas moves outward to form an outflow (Keto 2007). In this picture, the accretion is confined to a narrow range of angles close to the mid-plane of the disk and is close to termination. Without the observational evidence of the gas dynamics in the inner most region of $\mathrm{UC} 1$, the accretion status of $\mathrm{UC} 1$ is controversial.

Because of the large inclination angle of IRS5A from the plane of the sky, near-IR emission lines produced in the accretion flow (if exists) can be seen without the severe attenuation by the disk. In fact, IRS5A does not show any emission line indicating accretion. We have proposed two reasons for the absence of accretion indicators in Sect. 5.3. If in the case of the nonaccretion phase, the evolutionary stage of IRS5A would imply that accretion could even be halted before high-mass protostar arriving on ZAMS. In view of the lower temperature at the early stage of high-mass protostar, radiation feedback is not as strong as for a ZAMS star, thus outflow activities are additionally required to be responsible for the reversed accretion. In the large picture, the expanding $\mathrm{H}$ II region of M 17 might also contribute to the dissipation of accretion tunnel flows. The deduced timescale of accretion phase is thus $\sim 1 \times 10^{5} \mathrm{yrs}$, on the same order of high-mass protostar's age. On the other hand, the probable periodic accretion process of IRS5A would suggest a much longer timescale of accretion phase. The determination of the gas dynamics in the inner most area of IRS5A will disentangle this confusing puzzle.

Interestingly, the absence of hydrogen recombination lines in B273A's spectrum implies a terminated-accretion phase. An intermediate-mass YSO with an age $\sim 4 \times 10^{5}$ yrs like B273A is most likely to show accretion activity because YSOs at later evolutionary stages, such as intermediate-mass $\mathrm{T}$ Tauri stars (1.5-4 $\left.M_{\odot}, 1-10 \mathrm{Myr}\right)$, were observed to show $\mathrm{Br} \gamma$ emission even in low-resolution $(R \sim 800)$ near-IR spectra (Calvet et al. $2004)$. This discrepancy between the observation and the predication for B273A indicates additional feedback to dissipate the accretion funnel flows. The exact spatial coincident between B273A and the IF implies that the expanding $\mathrm{H}$ II region driven by the ionizing photons is the plausible mechanism of external feedback.

From the current masses and ages of these two objects, IRS5A and B273A require mass accretion rate of $9 \times$ $10^{-5} M_{\odot} \mathrm{yr}^{-1}$ and $1 \times 10^{-5} M_{\odot} \mathrm{yr}^{-1}$, respectively. Even in the possibility of weak $\operatorname{Br} \gamma$, we still note a dramatically drop of $\dot{M}$ for both objects. This could be also the result of feedback from stellar activities and expanding $\mathrm{H}$ II region. For the two low-mass YSOs, B273B and IRS5B, we could similarly estimate that the upper limit of their mass accretion rates is definitely lower than that of B273A, i.e., $\sim 3.8 \times 10^{-8} M_{\odot} \mathrm{yr}^{-1}$. The typical mass accretion rate of low-mass YSOs is on the order of $1 \times 10^{-8} M_{\odot} \mathrm{yr}^{-1}$ (e.g., Fang et al. 2013; Alcalá et al. 2014), which is comparable to the detection limit of B273B and IRS5B's spectra. The 
lack of $\mathrm{Br} \gamma$ emission line in the spectra of low-mass YSOs (e.g., B273B, IRS5B) is reminiscent of the nonaccretion phase, however, we cannot rule out the possibility that their accretion indicators could be revealed by higher resolution spectroscopic observation. In the case of the nonaccretion phase for these lowmass YSOs, external radiation feedback might be important in the disk clearing process for the YSOs in massive star-forming regions.

\subsection{Properties of M17 SW}

Previous studies of M 17 SW speculated that a dense PDR with high-density clumps and low-density gas matched the observed properties of M 17 SW (Meixner et al. 1992; Pérez-Beaupuits et al. 2010, 2012). However, these studies are based on farIR and radio line observations, and thus cannot resolve structures smaller than several arcseconds. The near-IR $\mathrm{H}_{2}$ emission detected in the M $17 \mathrm{UC1}$ - IRS5 region with extremely high spatial resolution (100 mas) set a entirely new picture for the warmest part of M $17 \mathrm{SW}$. The column densities of several $\mathrm{H}_{2} v=1-0$ ro-vibrational lines show that $\mathrm{H}_{2}$ gas mostly concentrates in filaments in the range $0.02-0.05 \mathrm{pc}$. The $\mathrm{H}_{2}$ line ratios, as we discussed in Sect. 4.3.3, refer to a gas density of $>10^{5} \mathrm{~cm}^{-3}$ if they are compared to PDR models (Burton et al. 1990). The high-density nature of M 17 SW is also evidenced by the molecular gas persisting just west of the IF (Pérez-Beaupuits et al. 2012). This can be interpreted as the result of self-shielding of molecules in high-density PDR, which can move the transition regions for $\mathrm{HI} / \mathrm{H}_{2}$ close to the surface of the cloud (Burton et al. 1990). Complementally, the $\mathrm{H}_{2}$ gas temperature characterized by the ro-vibrational diagram is in the range $500-750 \mathrm{~K}$. In contrast, H I observations toward M 17 specifically showed that H I number density toward the M 17 UC1 - IRS5 region is in the range $3.7 \times 10^{3}-1.5 \times 10^{4} \mathrm{~cm}^{-3}$, which corresponds to H I spin temperature between $50 \mathrm{~K}$ and $200 \mathrm{~K}$ in many directions toward M 17 (Brogan et al. 1999). Therefore, the $\mathrm{H}_{2}$ filaments presented here confirm for the first time that dense clumps with a number density two orders of magnitude higher and temperatures higher than ambient atomic gas can exist down to $10^{-2}$ pc scale inside the dense PDR M 17 SW, which was previously considered to be clumpy (Meixner et al. 1992).

\section{Summary and conclusions}

We presented diffraction-limited near- to mid-IR images and SINFONI integral field spectroscopy at $H+K$ toward the M 17 UC1 - IRS5 region, which shows emission lines of He I, H I, and $\mathrm{H}_{2}$. Our diffraction-limited data reveal new fine structures of this region.

This work complements the work by $\mathrm{CZ}+12$ from a view of much higher angular resolution. The IRN identified in $\mathrm{CZ}+12$ and illuminated by IRS5A and UC1 are confirmed to trace the molecular outflow driven by the two objects, respectively. Combining the SINFONI $\mathrm{H}_{2}$ line emission map and the midIR VISIR image, a blue-shifted outflow lobe is proposed for IRS5A, with two types of tracers in the form of $\mathrm{H}_{2}$ emission filament and mid-IR emission. The molecular outflow UC1 runs south-north with its blue-shifted lobe merely visible in forms of near-IR polarized light and mid-IR emission. The northeastern side of UC1's outflow is suppressed by the shock fronts driven by the expanding H II region. Thus a sheet of warm, dense gas forms at the northeastern edge of UC1's molecular outflow, and appears in forms of the bar-like emission seen in the $K$-band and $\mathrm{H}_{2}$ emission filament, which are both parallel to the ionization front.
The uniform line ratios of all $\mathrm{H}_{2}$ emitting areas indicate the same excitation mechanism; the $\mathrm{H}_{2}$ molecules are initially pumped by FUV photons, and are repopulated by the collisional de-excitation in a dense PDR. The $\mathrm{H}_{2}$ gas excitation temperature is estimated to be around $575 \mathrm{~K}$ based on the ro-vibrational diagram of the detected $\mathrm{H}_{2} v=1$ lines. The $\mathrm{H}_{2}$ line ratios are used to probe the properties of the PDR. Comparisons to PDR models suggest clumpy PDR structure with at least two components: warm, dense molecular clumps with $n_{\mathrm{H}}>10^{5} \mathrm{~cm}^{-3}$ and $T \sim 575 \mathrm{~K}$ and atomic gas with $n_{\mathrm{H}} \sim 3.7 \times 10^{3}-1.5 \times 10^{4} \mathrm{~cm}^{-3}$ and $T \sim 50-200 \mathrm{~K}$.

IRS5 might be a young quadruple system containing IRS5A, IRS5B, IRS5C, and IRS5D. Its primary star IRS5A is classified as a B3-B7 V/III star with a bolometric luminosity of 3.0-8.6 $\times$ $10^{3} L_{\odot}$, confirmed to be a high-mass protostar with mass $\sim 9 M_{\odot}$ and age $\sim 1 \times 10^{5} \mathrm{yrs}$, while the three lower mass companions are much less constrained. Particularly, IRS5C might be a deeply embedded high-mass protostellar object with a dusty disk. The spectral type of B273A is assigned to G4/5 III, suggesting an intermediate-mass YSO of $\sim 4 M_{\odot}$ and an age $\sim 4 \times 10^{5}$ yrs when compared with the nonaccretion PMS model. B273A may have a lower mass companion B273B of possibly similar age.

The Bry emission of UC1 is not clear enough to trace the accretion because the emission can arise from the HCHII region as well; UC1 might have terminated accretion from a concern on the ratio of $R_{\mathrm{i}} \gg R_{\mathrm{b}}$. The absence of $\mathrm{Br} \gamma$ emission in the SINFONI spectra of the other YSOs of various masses implies terminated accretion for them. Even in the case of weak $\mathrm{Br} \gamma$ emission that cannot be resolved by the SINFONI data, the steep drop in the accretion rates of IRS5A and B273A along the protostellar evolution suggests processes of dissipating accretion funnel flows. The object IRS5A is unique because it is during a stage when its radiation feedback is not high enough to severely destroy accretion funnel flows or even to terminate the accretion; therefore, we suggest that both the expanding $\mathrm{H}$ II region and its bipolar outflow contribute simultaneously. For the other YSOs, the expanding H II region could be the major mechanism on dissipating/terminating accretion.

Acknowledgements. This work is supported by the Strategic Priority Research Program "The Emergence of Cosmological Structure" of the Chinese Academy of Sciences, grant No. XDB09000000. Z.J. acknowledges the support by NSFC 11233007. M.F. acknowledges the support by the NSFC through grants 11203081. Z.C. acknowledges Dr. S. Zhang for his IDL routines to make the RGB figures look good. This publication used data products from the Two Micron All Sky Survey, which is a joint project of the University of Massachusetts and the Infrared Processing and Analysis Center/California Institute of Technology, funded by the National Aeronautics and Space Administration and the National Science Foundation. This research made use of the SIMBAD database, operated at the CDS, Strasbourg, France. This research made use of NASA's Astrophysics Data System Bibliographic Services.

\section{References}

Alcalá, J. M., Natta, A., Manara, C. F., et al. 2014, A\&A, 561, A2

Allers, K. N., Jaffe, D. T., Lacy, J. H., Draine, B. T., \& Richter, M. J. 2005, ApJ, 630,368

Bik, A., Kaper, L., Hanson, M. M., \& Smits, M. 2005, A\&A, 440, 121

Bik, A., Puga, E., Waters, L. B. F. M., et al. 2010, ApJ, 713, 883

Blum, R. D., \& McGregor, P. J. 2008, AJ, 135, 1708

Breen, S. L., Caswell, J. L., Ellingsen, S. P., \& Phillips, C. J. 2010, MNRAS, 406, 1487

Brogan, C. L., \& Troland, T. H. 2001, ApJ, 560, 821

Brogan, C. L., Troland, T. H., Roberts, D. A., \& Crutcher, R. M. 1999, ApJ, 515 , 304

Burton, M. G., Geballe, T. R., Brand, P. W. J. L., \& Moorhouse, A. 1990, ApJ, 352,625

Calvet, N., Muzerolle, J., Briceño, C., et al. 2004, AJ, 128, 1294

Cardelli, J. A., Clayton, G. C., \& Mathis, J. S. 1989, ApJ, 345, 245 


\section{Z. Chen et al.: VLT IR study of M 17 UC1 - IRS5 region}

Carmona, A., van der Plas, G., van den Ancker, M. E., et al. 2011, A\&A, 533, A39

Casali, M. M., \& Eiroa, C. 1996, A\&A, 306, 427

Caswell, J. L. 1998, MNRAS, 297, 215

Caswell, J. L., Vaile, R. A., Ellingsen, S. P., Whiteoak, J. B., \& Norris, R. P. 1995, MNRAS, 272, 96

Caswell, J. L., Yi, J., Booth, R. S., \& Cragg, D. M. 2000, MNRAS, 313, 599

Chapman, N. L., Mundy, L. G., Lai, S.-P., \& Evans, II, N. J. 2009, ApJ, 690, 496

Chen, Z., Jiang, Z., Wang, Y., et al. 2012, PASJ, 64, 110 (CZ+12)

Chen, Z., Nürnberger, D. E. A., Chini, R., et al. 2013, A\&A, 557, A51

Chini, R., Nielbock, M., \& Beck, R. 2000, A\&A, 357, L33

Churchwell, E. 2002, ARA\&A, 40, 27

Claret, A. 2012, A\&A, 541, A113

Cox, A. N. 2000, Allen's astrophysical quantities, 100, 72

Dabrowski, I. 1984, Can. J. Phys., 62, 1639

Ducati, J. R., Bevilacqua, C. M., Rembold, S. B., \& Ribeiro, D. 2001, ApJ, 558, 309

Fang, M., van Boekel, R., Bouwman, J., et al. 2013, A\&A, 549, A15

Fontani, F., Cesaroni, R., \& Furuya, R. S. 2010, A\&A, 517, A56

Gatley, I., Hasegawa, T., Suzuki, H., et al. 1987, ApJ, 318, L73

Gillessen, S., Davies, R., Kissler-Patig, M., et al. 2005, The Messenger, 120, 26

González-Fernández, C., \& Negueruela, I. 2012, A\&A, 539, A100

Grave, J. M. C., \& Kumar, M. S. N. 2009, A\&A, 498, 147

Hanson, M. M., Conti, P. S., \& Rieke, M. J. 1996, ApJS, 107, 281

Hanson, M. M., Howarth, I. D., \& Conti, P. S. 1997, ApJ, 489, 698

Hanson, M. M., Rieke, G. H., \& Luhman, K. L. 1998, AJ, 116, 1915

Hanson, M. M., Kudritzki, R.-P., Kenworthy, M. A., Puls, J., \& Tokunaga, A. T. 2005, ApJS, 161, 154

Hatch, N. A., Crawford, C. S., Fabian, A. C., \& Johnstone, R. M. 2005, MNRAS, 358,765

Hayashi, M., Hasegawa, T., Gatley, I., Garden, R., \& Kaifu, N. 1985, MNRAS, $215,31 \mathrm{P}$

Hoffmeister, V. H. 2008, Ph.D. Thesis, Ruhr-Universität Bochum

Hoffmeister, V. H., Chini, R., Scheyda, C. M., et al. 2008, ApJ, 686, 310

Hoogerwerf, R., de Bruijne, J. H. J., \& de Zeeuw, P. T. 2000, ApJ, 544, L133

Hosokawa, T., \& Omukai, K. 2009, ApJ, 691, 823

Hosokawa, T., Yorke, H. W., \& Omukai, K. 2010, ApJ, 721, 478 (H+10)

Hosokawa, T., Offner, S. S. R., \& Krumholz, M. R. 2011, ApJ, 738, 140

Johnson, C. O., Depree, C. G., \& Goss, W. M. 1998, ApJ, 500, 302

Kassis, M., Deutsch, L. K., Campbell, M. F., et al. 2002, AJ, 124, $1636(\mathrm{~K}+02)$

Keto, E. 2007, ApJ, 666, 976

Kumar, M. S. N., \& Grave, J. M. C. 2007, A\&A, 472, 155

Lada, C. J., \& Adams, F. C. 1992, ApJ, 393, 278

Lagage, P. O., Pel, J. W., Authier, M., et al. 2004, The Messenger, 117, 12

Lenzen, R., Hartung, M., Brandner, W., et al. 2003, in Instrument Design and Performance for Optical/Infrared Ground-based Telescopes, eds. M. Iye, \& A. F. M. Moorwood, SPIE Conf. Ser., 4841, 944
Longmore, S. N., Pillai, T., Keto, E., Zhang, Q., \& Qiu, K. 2011, ApJ, 726, 97 Luhman, K. L., Engelbracht, C. W., \& Luhman, M. L. 1998, ApJ, 499, 799 Lumsden, S. L., Puxley, P. J., \& Hoare, M. G. 2001, MNRAS, 328, 419

Martín-Hernández, N. L., Bik, A., Puga, E., Nürnberger, D. E. A., \& Bronfman, L. 2008, A\&A, 489, 229

Meixner, M., \& Tielens, A. G. G. M. 1993, ApJ, 405, 216

Meixner, M., Haas, M. R., Tielens, A. G. G. M., Erickson, E. F., \& Werner, M. 1992, ApJ, 390, 499

Mendigutía, I., Calvet, N., Montesinos, B., et al. 2011, A\&A, 535, A99

Meyer, M. R., Calvet, N., \& Hillenbrand, L. A. 1997, AJ, 114, 288

Molinari, S., Pezzuto, S., Cesaroni, R., et al. 2008, A\&A, 481, 345

Murphy, T., Cohen, M., Ekers, R. D., et al. 2010, MNRAS, 405, 1560

Nielbock, M., Chini, R., Jütte, M., \& Manthey, E. 2001, A\&A, 377, 273 (N+01)

Nielbock, M., Chini, R., Hoffmeister, V. H., et al. 2007, ApJ, 656, L81 (N+07)

Nürnberger, D. E. A., Chini, R., Eisenhauer, F., et al. 2007, A\&A, 465, 931

Okumura, S.-I., Mori, A., \& Yanagisawa, K. 2004, PASJ, 56, 705

Pecaut, M. J., \& Mamajek, E. E. 2013, ApJS, 208, 9

Pellegrini, E. W., Baldwin, J. A., Brogan, C. L., et al. 2007, ApJ, 658, 1119

Pérez-Beaupuits, J. P., Spaans, M., Hogerheijde, M. R., et al. 2010, A\&A, 510, A87

Pérez-Beaupuits, J. P., Wiesemeyer, H., Ossenkopf, V., et al. 2012, A\&A, 542, L13

Rigliaco, E., Natta, A., Testi, L., et al. 2012, A\&A, 548, A56

Robitaille, T. P., Whitney, B. A., Indebetouw, R., \& Wood, K. 2007, ApJS, 169, 328

Roche, P. F., \& Aitken, D. K. 1985, MNRAS, 215, 425

Rousset, G., Lacombe, F., Puget, P., et al. 2003, in Adaptive Optical System Technologies II, eds. P. L. Wizinowich, \& D. Bonaccini, SPIE Conf. Ser., 4839, 140

Sewilo, M., Churchwell, E., Kurtz, S., Goss, W. M., \& Hofner, P. 2004, ApJ, 605,285

Sheffer, Y., \& Wolfire, M. G. 2013, ApJ, 774, L14

Sternberg, A., \& Dalgarno, A. 1989, ApJ, 338, 197

Stutzki, J., \& Guesten, R. 1990, ApJ, 356, 513

Stutzki, J., Stacey, G. J., Genzel, R., et al. 1988, ApJ, 332, 379

Tanaka, M., Hasegawa, T., Hayashi, S. S., Brand, P. W. J. L., \& Gatley, I. 1989, ApJ, 336, 207

Torrelles, J. M., Trinidad, M. A., Curiel, S., et al. 2014, MNRAS, 437, 3803

Turner, J., Kirby-Docken, K., \& Dalgarno, A. 1977, ApJS, 35, 281

Wallace, L., \& Hinkle, K. 1997, ApJS, 111, 445

Whittet, D. C. B. 2003, Dust in the galactic environment (Bristol: Institute of Physics (IOP) Publishing)

Xu, Y., Moscadelli, L., Reid, M. J., et al. 2011, ApJ, 733, 25

Yorke, H. W., \& Bodenheimer, P. 2008, in Massive Star Formation: Observations Confront, Theory, eds. H. Beuther, H. Linz, \& T. Henning, ASP Conf. Ser., 387,189

Zinnecker, H., \& Yorke, H. W. 2007, ARA\&A, 45, 481 